Annales Fennici Mathematici

Volumen 47, 2022, 57-82

\title{
VMO-Teichmüller space on the real line
}

\author{
YULIANG SHEN
}

\begin{abstract}
An increasing homeomorphism $h$ on the real line $\mathbb{R}$ is said to be strongly symmetric if it can be extended to a quasiconformal homeomorphism of the upper half plane $\mathbb{U}$ onto itself whose Beltrami coefficient $\mu$ induces a vanishing Carleson measure $|\mu(z)|^{2} / y d x d y$ on $\mathbb{U}$. We will deal with the class of strongly symmetric homeomorphisms on the real line and its Teichmüller space, which we call the VMO-Teichmüller space. In particular, we will show that if $h$ is strongly symmetric on the real line, then it is strongly quasisymmetric such that $\log h^{\prime}$ is a VMO function. This improves some classical results of Carleson (1967) and Anderson-Becker-Lesley (1988) on the problem about the local absolute continuity of a quasisymmetric homeomorphism in terms of the Beltrami coefficient of a quasiconformal extension. We will also discuss various models of the VMO-Teichmüller space and endow it with a complex Banach manifold structure via the standard Bers embedding.
\end{abstract}

\section{Reaaliakselin VMO-Teichmüllerin avaruus}

Tiivistelmä. Reaaliakselin kasvavaa homeomorfismia $h$ sanotaan vahvasti symmetriseksi, jos se voidaan jatkaa ylemmän puolitason $\mathbb{U}$ itselleen kuvaavaksi kvasikonformiseksi homeomorfismiksi, jonka Beltramin kerroin $\mu$ määrittelee puolitason $\mathbb{U}$ häviävän Carlesonin mitan $|\mu(z)|^{2} / y d x d y$. Tarkastelemme reaaliakselin vahvasti symmetristen homeomorfismien luokkaa ja sen Teichmüllerin avaruutta, jota kutsumme VMO-Teichmüllerin avaruudeksi. Osoitamme erityisesti, että jos $h$ on vahvasti symmetrinen reaaliakselilla, niin se on vahvasti kvasisymmetrinen ja log $h^{\prime}$ on VMO-funktio. Tämä parantaa eräitä Carlesonin (1967) sekä Andersonin, Beckerin ja Lesleyn (1988) klassisia tuloksia, jotka käsittelevät kvasisymmetrisen homeomorfismin lokaalin absoluuttisen jatkuvuuden ongelmaa sen kvasikonformisen jatkeen Beltramin kertoimen kautta. Käsittelemme myös useita VMOTeichmüllerin avaruuden malleja ja annamme sille standardin Bersin upotuksen kautta kompleksisen Banachin moniston rakenteen.

\section{Introduction}

We first fix some basic notations. Let $\mathbb{U}=\{z=x+i y: y>0\}$ and $\mathbb{U}^{*}=$ $\{z=x+i y: y<0\}$ denote the upper and lower half plane in the complex plane $\mathbb{C}$, respectively. $\mathbb{R}=\partial \mathbb{U}=\partial \mathbb{U}^{*}$ is the real line, and $\hat{\mathbb{R}}=\mathbb{R} \cup\{\infty\}$ is the extended real line in the Riemann sphere $\hat{\mathbb{C}}=\mathbb{C} \cup\{\infty\}$. Let $\Delta=\{z:|z|<1\}$ denote the unit disk. $\Delta^{*}=\hat{\mathbb{C}}-\bar{\Delta}$ is the exterior of $\Delta$, and $S^{1}=\partial \Delta=\partial \Delta^{*}$ is the unit circle.

Let $\operatorname{Hom}^{+}(\mathbb{R})$ denote the group of all increasing homeomorphisms of $\mathbb{R}$ onto itself. A homeomorphism $h \in \mathrm{Hom}^{+}(\mathbb{R})$ is said to be quasisymmetric if there exists some

https://doi.org/10.54330/afm.112456

2020 Mathematics Subject Classification: Primary 30C62; Secondary 30D50, 30F60, 32G15.

Key words: Universal Teichmüller space, quasiconformal mapping, quasisymmetric homeomorphism, Beltrami coefficient, strongly symmetric homeomorphism, Carleson measure, vanishing Carleson measure, BMOA, VMOA.

Research supported by the National Natural Science Foundation of China (Grant Nos. $11631010,12171346)$.

(c) 2022 The Finnish Mathematical Society 
constant $M(h)>0$ such that

$$
\frac{1}{M(h)} \leq \frac{h(x+t)-h(x)}{h(x)-h(x-t))} \leq M(h)
$$

for all $x \in \mathbb{R}$ and $t>0$. The notion of quasisymmetric homeomorphism was first introduced by Beurling-Ahlfors [BA], who also proved that $h \in \operatorname{Hom}^{+}(\mathbb{R})$ is quasisymmetric if and only if there exists some quasiconformal homeomorphism of $\mathbb{U}$ onto itself which has boundary values $h$.

The universal Teichmüller space $T$ is a universal parameter space for all Riemann surfaces and can be defined as the space of all normalized quasisymmetric homeomorphisms on the real line, namely, $T=\mathrm{QS}(\mathbb{R}) / \operatorname{Aff}(\mathbb{R})$. Here, $\mathrm{QS}(\mathbb{R})$ denotes the group of all quasisymmetric homeomorphisms of the real line, and $A f f(\mathbb{R})$ the subgroup of all real affine mappings $z \mapsto a z+b, a>0, b \in \mathbb{R}$. It is well known that the universal Teichmüller space $T$ is an infinite dimensional complex Banach manifold, and $\mathrm{QS}(\mathbb{R})$ has a smooth Banach manifold structure such that QS $(\mathbb{R})$ is diffeomorphic to $T \times \operatorname{Aff}(\mathbb{R})($ see $[\mathrm{Ga}],[\mathrm{GL}],[\mathrm{Le}],[\mathrm{Na}])$.

A quasisymmetric homeomorphism $h$ is said to be symmetric if

$$
\lim _{t \rightarrow 0+} \frac{h(x+t)-h(x)}{h(x)-h(x-t))}=1
$$

uniformly for all $x \in \mathbb{R}$. Let $\mathrm{S}(\mathbb{R})$ denote the set of all symmetric homeomorphisms of the real line. This class was first studied in [Ca] when Carleson discussed the absolute continuity of a quasisymmetric homeomorphism. It was investigated in depth later by Gardiner-Sullivan [GS] during their study of little Teichmüller spaces and asymptotic Teichmüller spaces. In particular, it was proved that a quasisymmetric homeomorphism $h$ is symmetric if and only if $h$ can be extended as an asymptotically conformal mapping $f$ to the upper half plane, and that the Beurling-Ahlfors extension of $h$ is asymptotically conformal when $h$ is symmetric (see [Ca], [GS], [Mat]). Here by an asymptotically conformal mapping $f$ of the upper half plane onto itself we mean that its complex dilatation $\mu=\bar{\partial} f / \partial f$ satisfies the condition $\mu(x+i y) \rightarrow 0$ uniformly for all $x \in \mathbb{R}$ when $y \rightarrow 0+$. In a recent paper [HWS] we showed that $T_{0}=\mathrm{S}(\mathbb{R}) / \operatorname{Aff}(\mathbb{R})$, which we call the symmetric Teichmüller space, can be endowed with a complex Banach manifold structure. More recently, Wei-Matsuzaki [WM1] further generalized and discussed the class of symmetric homeomorphisms in the setting of the unit circle.

In this paper, we will introduce and discuss a new subclass of quasisymmetric homeomorphisms, which we call strongly symmetric homeomorphisms. We first recall the notion of strongly quasisymmetric homeomorphism in the sense of Semmes [Se2]. A homeomorphism $h \in \mathrm{Hom}^{+}(\mathbb{R})$ is said to be strongly quasisymmetric if there exist two positive constants $C_{1}(h), C_{2}(h)$ such that

$$
\frac{|h(E)|}{|h(I)|} \leq C_{1}(h)\left(\frac{|E|}{|I|}\right)^{C_{2}(h)}
$$

whenever $I \subset \mathbb{R}$ is an interval and $E \subset I$ a measurable subset. In other words, $h$ is strongly quasisymmetric if and only if $h$ is locally absolutely continuous so that $h^{\prime}$ belongs to the class of weights $A^{\infty}$ introduced by Muckenhoupt (see [CF], [Gar]), in particular, $\log h^{\prime}$ belongs to $\operatorname{BMO}(\mathbb{R})$, the space of locally integrable functions on $\mathbb{R}$ of bounded mean oscillation (see [FS], [Gar] and section 8 below). Let $\operatorname{SQS}(\mathbb{R})$ denote the set of all strongly quasisymmetric homeomorphisms of $\mathbb{R}$ onto itself. Then $\operatorname{SQS}(\mathbb{R})$ is a sub-group of $\mathrm{QS}(\mathbb{R})$ and $T_{b}=\operatorname{SQS}(\mathbb{R}) / \operatorname{Aff}(\mathbb{R})$ is called the 
BMO-Teichmüller space. This sub-class of quasisymmetric homeomorphisms and its Teichmüller space were much investigated (see [AZ], [CZ], [FHS1-2], [FKP], [HS], [Ma], [Se2], [SW], [TWS], [We], [WM2], [WS], [WZ]) because of their great importance in the application to harmonic analysis and elliptic operator theory (see [Da], $[\mathrm{FKP}],[\mathrm{Jo}]$, [Se1]). In particular, it was proved in [FKP] (see also [Se2] and [AZ], [Ma]) that a homeomorphism $h \in \mathrm{Hom}^{+}(\mathbb{R})$ is strongly quasisymmetric if and only if it can be extended to a quasiconformal homeomorphism of $\mathbb{U}$ onto itself whose Beltrami coefficient $\mu$ induces a Carleson measure $|\mu(z)|^{2} / y d x d y$ on the upper half plane (see $\S 2$ for precise definition). In [SW] we proved that $T_{b}$ is an infinite dimensional complex Banach manifold.

Now we say that a homeomorphism $h \in \mathrm{Hom}^{+}(\mathbb{R})$ is strongly symmetric if it can be extended to a quasiconformal homeomorphism of $\mathbb{U}$ onto itself whose Beltrami coefficient $\mu$ induces a vanishing Carleson measure $|\mu(z)|^{2} / y d x d y$ on the upper half plane (see $\S 2$ for precise definition). Let $S S(\mathbb{R})$ denote the set of all strongly symmetric homeomorphisms of the real line. As a subclass of $\operatorname{SQS}(\mathbb{R}), \operatorname{SS}(\mathbb{R})$ is much related with and also has wide application to some important problems in real and harmonic analysis (see $[\mathrm{Da}]$ ). As it will be shown later, $\mathrm{SS}(\mathbb{R})$ is also a subclass of $S(\mathbb{R})$, namely, a strongly symmetric homeomorphism must be symmetric (see Theorem 4.2 below), which can not be deduced directly just by definition.

In this paper, we will give some basic properties of a strongly symmetric homeomorphism and endow $T_{v}=\mathrm{SS}(\mathbb{R}) / \operatorname{Aff}(\mathbb{R})$, which we call the VMO-Teichmüller space, with a complex Banach manifold structure under which $T_{v}$ can be biholomorphically embedded as a bounded domain in certain Banach space (see Theorem 2.1 below). We will also discuss various properties of some other models of the VMO-Teichmüller space $T_{v}$ in sections 7 and 8 (see Theorems 7.5, 8.2 below).

Remark 1.1. The BMO-Teichmüller space $T_{b}$ is usually defined and discussed on the unit circle $S^{1}$ (see [AZ], [SW]). In this paper, we consider the real line case and use strongly quasisymmetric homeomorphisms on the real line to define the BMOTeichmüller space $T_{b}$ (see [FSH2]). Due to the conformal invariance of Carleson measures and strongly quasisymmetric homeomorphisms, we have a parallel theory of the BMO-Teichmüller space $T_{b}$ of the real line model to that of the unit circle model, which was developed in the papers [AZ] and [SW].

Remark 1.2. A sense preserving homeomorphism $g$ on the unit circle $S^{1}$ is called strongly symmetric if it is absolutely continuous such that $\log g^{\prime}$ belongs to $\operatorname{VMO}\left(S^{1}\right)$, the space of integrable functions on $S^{1}$ of vanishing mean oscillation (see [Gar], [Sa] and section 8 below), a condition which first appeared in the paper [Pa] where Partyka studied the so-called eigenvalues of a quasicircle. We discussed the class of strongly symmetric homeomorphisms on the unit circle in the paper [SW] and proved particularly there that $g$ is strongly symmetric if and only if it can be extended to a quasiconformal homeomorphism of $\Delta$ onto itself whose Beltrami coefficient $\nu$ induces a vanishing Carleson measure $|\nu(z)|^{2} /\left(1-|z|^{2}\right) d x d y$ on the unit disk. This inspires us to introduce in the paper the new concept of strongly symmetric homeomorphism on the real line. Since the vanishing Carleson measure does not have conformal invariance, the notion of strongly symmetric homeomorphism on the real line does not correspond to the one on the unit circle. Actually, it is not known how to characterize a strongly symmetric homeomorphism on the real line without using quasiconformal extensions. In section 8, we will show that if $h \in \operatorname{Hom}^{+}(\mathbb{R})$ is strongly symmetric, then it is strongly quasisymmetric such that $\log h^{\prime} \in \operatorname{VMO}(\mathbb{R})$, 
the space of locally integrable functions on $\mathbb{R}$ of vanishing mean oscillation (see [Gar], [Sa] and section 8 below). This improves some classical results of Carleson [Ca] and Anderson-Becker-Lesley [ABL] on the open problem concerning the local absolute continuity of a quasisymmetric homeomorphism in terms of the Beltrami coefficient of a quasiconformal extension.

Notations. $C, C_{1}, C_{2} \cdots$ will denote universal constants that might change from one line to another, while $C(\cdot), C_{1}(\cdot), C_{2}(\cdot) \cdots$ will denote constants that depend only on the elements put in the brackets. The notation $A \lesssim B(A \gtrsim B)$ means that there is a positive constant $C$ independent of $A$ and $B$ such that $A \leq C B(A \geq C B)$. The notation $A \asymp B$ means both $A \lesssim B$ and $A \gtrsim B$.

\section{Preliminaries and statement of results}

In this section, we recall some basic definitions and results on (BMO) Teichmüller theory and state some results of the paper. For primary references, see GardinerLakic [GL], Garnett [Gar], Lehto [Le] and Nag [Na].

2.1. Universal Teichmüller space. Let $M(\mathbb{U})$ denote the open unit ball of the Banach space $L^{\infty}(\mathbb{U})$ of essentially bounded measurable functions on the upper half plane $\mathbb{U}$. For $\mu \in M(\mathbb{U})$, let $f^{\mu}$ be the unique quasiconformal mapping on $\mathbb{U}$ onto itself which has complex dilatation $\mu$ and keeps the points 0,1 and $\infty$ fixed. We say two elements $\mu$ and $\nu$ in $M(\mathbb{U})$ are equivalent, denoted by $\mu \sim \nu$, if $f^{\mu}=f^{\nu}$ on the real line $\mathbb{R}$. We let $[\mu]$ denote the equivalence class of $\mu$. Then the correspondence $\left.[\mu] \mapsto f^{\mu}\right|_{\mathbb{R}}$ establishes a one-to-one map from $M(\mathbb{U}) / \sim$ onto the universal Teichmüller space $T$. $T=M(\mathbb{U}) / \sim$ is known as the Bers model of the universal Teichmüller space. We let $\Phi$ denote the natural projection from $M(\mathbb{U})$ onto $T$ so that $\Phi(\mu)$ is the equivalence class $[\mu]$. [0] is called the base point of $T$.

It is known that the universal Teichmüller space $T$ is an infinite dimensional complex Banach manifold. To make this precise, we need to define the so-called Bers projection and Bers embedding. Let $\Omega$ be an arbitrary simply connected domain in the extended complex plane $\hat{\mathbb{C}}$ which is conformally equivalent to the upper half plane. The hyperbolic metric $\lambda_{\Omega}$ on $\Omega$ can be defined by

$$
\lambda_{\Omega}(f(z))\left|f^{\prime}(z)\right|=\frac{1}{y}, \quad z=x+i y \in \mathbb{U},
$$

where $f: \mathbb{U} \rightarrow \Omega$ is any conformal mapping. Let $B(\Omega)$ denote the Banach space of functions $\phi$ holomorphic in $\Omega$ with norm

$$
\|\phi\|_{B(\Omega)}=\sup _{z \in \Omega}|\phi(z)| \lambda_{\Omega}^{-2}(z) .
$$

Then the Bers projection is the map $\mathcal{S}: M(\mathbb{U}) \rightarrow B\left(\mathbb{U}^{*}\right)$ defined as $\mathcal{S}(\mu)=S_{\left.f_{\mu}\right|_{\mathbb{U}^{*}}}$, where $f_{\mu}$ is the unique quasiconformal mapping of the complex plane $\mathbb{C}$ which has complex dilatation $\mu$ in $\mathbb{U}$, is conformal in $\mathbb{U}^{*}$, and keeps the points 0,1 and $\infty$ fixed, while $S_{f}$ is the Schwarzian derivative of a locally univalent function $f$ of a domain in the extended plane $\hat{\mathbb{C}}$, defined as

$$
S_{f}=N_{f}^{\prime}-\frac{1}{2} N_{f}^{2}, \quad N_{f}=\frac{f^{\prime \prime}}{f^{\prime}} .
$$

It is known that $\mathcal{S}$ is a holomorphic split submersion and descends down to a one-toone map $\beta: T \rightarrow B\left(\mathbb{U}^{*}\right)$, which is known as the Bers embedding. Via the Bers embedding, $T$ carries a natural Banach manifold complex structure so that the natural 
projection $\Phi: M \rightarrow T$ is a holomorphic split submersion, and $\beta$ is a biholomorphism from $T$ onto its image.

Let $L_{0}(\mathbb{U})$ be the closed subspace of $L^{\infty}(\mathbb{U})$ which consists of those functions $\mu$ such that $\mu(x+i y) \rightarrow 0$ uniformly for $x \in \mathbb{R}$ as $y \rightarrow 0+$, or more precisely, for each $\epsilon>0$ there exists some $\delta>0$ such that $|\mu(z)|<\epsilon$ for almost every $z=x+i y$ with $0<y<\delta$. We denote by $B_{0}\left(\mathbb{U}^{*}\right)$ the closed subspace of $B\left(\mathbb{U}^{*}\right)$ which consists of those functions $\phi$ such that $y^{2} \phi(x+i y) \rightarrow 0$ uniformly for $x \in \mathbb{R}$ as $y \rightarrow 0$-. Set $M_{0}(\mathbb{U})=M(\mathbb{U}) \cap L_{0}(\mathbb{U})$. Then the correspondence $\left.[\mu] \mapsto f^{\mu}\right|_{\mathbb{R}}$ establishes a one-to-one map from $M_{0}(\mathbb{U}) / \sim$ onto the symmetric Teichmüller space $T_{0}$ (see [Ca], [GS]). In [HWS] (see also [WM1]) we proved that $\mathcal{S}$ maps $M_{0}(\mathbb{U})$ into $B_{0}\left(\mathbb{U}^{*}\right)$ and is a holomorphic split submersion from $M_{0}(\mathbb{U})$ onto its image, which implies that $T_{0}$ has a unique complex structure such that the natural projection $\Phi$ from $M_{0}(\mathbb{U})$ onto $T_{0}$ is a holomorphic split submersion.

2.2. (Vanishing) Carleson measure and BMO (VMO) Teichmüller space. Let $\lambda$ be a positive measure on the upper half plane $\mathbb{U}$. For $x \in \mathbb{R}$ and $h>0$, set

$$
Q=Q(x, h)=\left\{\zeta=\xi+i \eta: x-\frac{h}{2}<\xi<x+\frac{h}{2}, 0<\eta<h\right\} .
$$

Consider

$$
N_{\delta}(\lambda)=\sup \left\{\frac{\lambda(Q(x, h))}{h}: x \in \mathbb{R}, h<\delta\right\}
$$

and let

$$
N(\lambda)=\lim _{\delta \rightarrow+\infty} N_{\delta}(\lambda)
$$

and

$$
N_{0}(\lambda)=\lim _{\delta \rightarrow 0+} N_{\delta}(\lambda) .
$$

Then $\lambda$ is called a Carleson measure if $N(\lambda)<+\infty$. A Carleson measure $\lambda$ is called a vanishing Carleson measure if $N_{0}(\lambda)=0$. We denote by $C M(\mathbb{U})$ and $C M_{0}(\mathbb{U})$ the set of all Carleson measures and vanishing Carleson measures on $\mathbb{U}$, respectively. We can define $C M\left(\mathbb{U}^{*}\right)$ and $C M_{0}\left(\mathbb{U}^{*}\right)$ by the same way.

We denote by $\mathcal{L}(\mathbb{U})$ the Banach space of all essentially bounded measurable functions $\mu$ on $\mathbb{U}$ each of which induces a Carleson measure $\lambda_{\mu} \in C M(\mathbb{U})$ by $\lambda_{\mu}(z)=$ $|\mu(z)|^{2} / y d x d y, z=x+i y \in \mathbb{U}$. The norm on $\mathcal{L}(\mathbb{U})$ is defined as

$$
\|\mu\|_{c}=\|\mu\|_{\infty}+\sqrt{N\left(\lambda_{\mu}\right)} .
$$

$\mathcal{L}_{0}(\mathbb{U})$ is the closed subspace of $\mathcal{L}(\mathbb{U})$ consisting of all elements $\mu$ such that $\lambda_{\mu} \in$ $C M_{0}(\mathbb{U})$. Set $\mathcal{M}(\mathbb{U})=M(\mathbb{U}) \cap \mathcal{L}(\mathbb{U}), \mathcal{M}_{0}(\mathbb{U})=M(\mathbb{U}) \cap \mathcal{L}_{0}(\mathbb{U})$. Then $\mathcal{M}(\mathbb{U}) / \sim$ is the complex analytic model of the BMO-Teichmüller space $T_{b}$ (see $[\mathrm{FKP}]$ and also [AZ], [Ma], [Se2]), while by definition $\mathcal{M}_{0}(\mathbb{U}) / \sim$ is the one of the VMO-Teichmüller space $T_{v}$.

We denote by $\mathcal{B}\left(\mathbb{U}^{*}\right)$ the Banach space of functions $\phi$ holomorphic in $\mathbb{U}^{*}$ each of which induces a Carleson measure $\lambda_{\phi} \in C M\left(\mathbb{U}^{*}\right)$ by $\lambda_{\phi}(z)=|\phi(z)|^{2}|y|^{3} d x d y$, $z=x+i y \in \mathbb{U}^{*}$. The norm on $\mathcal{B}\left(\mathbb{U}^{*}\right)$ is

$$
\|\phi\|_{\mathcal{B}}=\sqrt{N\left(\lambda_{\phi}\right)}
$$


Lemma 7.1 below implies that $\mathcal{B}\left(\mathbb{U}^{*}\right) \subset B\left(\mathbb{U}^{*}\right)$, and the inclusion map is continuous. We denote by $\mathcal{B}_{0}\left(\mathbb{U}^{*}\right)$ the closed subspace of $\mathcal{B}\left(\mathbb{U}^{*}\right)$ consisting of all functions $\phi$ such that $\lambda_{\phi} \in C M_{0}\left(\mathbb{U}^{*}\right)$. Then $\mathcal{B}_{0}\left(\mathbb{U}^{*}\right) \subset B_{0}\left(\mathbb{U}^{*}\right)$ by Lemma 7.1 again.

Astala-Zinsmeister [AZ] (see also [BJ], [FKP], [Ma], [Se2] and Theorem 7.2 below) proved that $\mathcal{S}(\mathcal{M}(\mathbb{U}))=\mathcal{S}(M(\mathbb{U})) \cap \mathcal{B}\left(\mathbb{U}^{*}\right)$ under the Bers projection $\mathcal{S}$ : $M(\mathbb{U}) \rightarrow B\left(\mathbb{U}^{*}\right)$. Moreover, we proved in $[\mathrm{SW}]$ that $\mathcal{S}: \mathcal{M}(\mathbb{U}) \rightarrow \mathcal{B}\left(\mathbb{U}^{*}\right)$ is a holomorphic split submersion from $\mathcal{M}(\mathbb{U})$ onto its image, which implies that $T_{b}$ has a unique complex structure such that the natural projection $\Phi$ from $\mathcal{M}(\mathbb{U})$ onto $T_{b}$ is a holomorphic split submersion (see Remark 1.1).

2.3. Main results. In this paper, we will mainly discuss strongly symmetric homeomorphisms on the real line and the Bers projection $\mathcal{S}: M(\mathbb{U}) \rightarrow B\left(\mathbb{U}^{*}\right)$ on $\mathcal{M}_{0}(\mathbb{U})$. Our first main result is

Theorem 2.1. $\mathcal{S}$ maps $\mathcal{M}_{0}(\mathbb{U})$ onto a connected open subset in $\mathcal{B}_{0}\left(\mathbb{U}^{*}\right)$ and is holomorphic with local holomorphic sections. Consequently, $T_{v}$ has a unique complex structure such that $\beta: T_{v} \rightarrow \mathcal{B}_{0}\left(\mathbb{U}^{*}\right)$ is a bi-holomorphic map from $T_{v}$ onto a domain in $\mathcal{B}_{0}\left(\mathbb{U}^{*}\right)$. Under this complex structure, the natural projection $\Phi$ from $\mathcal{M}_{0}(\mathbb{U})$ onto $T_{v}$ is holomorphic with local holomorphic sections.

We will also discuss other models of the VMO-Teichmüller space in the latter part of the paper. The other main results (Theorems 7.5 and 8.2 below) are contained in the following

Theorem 2.2. Let $\mu \in M(\mathbb{U})$. Consider the following conditions:

(1) There exists some $\nu \in \mathcal{M}_{0}(\mathbb{U})$ such that $[\nu]=[\mu]$;

(2) $\left.\log f_{\mu}^{\prime}\right|_{\mathbb{U}^{*}} \in \operatorname{VMOA}\left(\mathbb{U}^{*}\right)$, the space of analytic functions in $\mathbb{U}^{*}$ of vanishing mean oscillation (see [FS], [Gar], [Sa]);

(3) $\mathcal{S}(\mu) \in \mathcal{B}_{0}\left(\mathbb{U}^{*}\right)$;

(4) $h=\left.f^{\mu}\right|_{\mathbb{R}}$ is strongly quasisymmetric such that $\log h^{\prime} \in \operatorname{VMO}(\mathbb{R})$.

Then $(1) \Rightarrow(2) \Leftrightarrow(3)$, and $(1) \Rightarrow(4)$.

Proof. $(1) \Rightarrow(3)$ follows from Proposition 5.1, (2) $\Leftrightarrow(3)$ from Theorem 7.5, and $(1) \Rightarrow$ (4) from Theorem 8.2.

\section{More on (vanishing) Carleson measures}

In this section we discuss some basic results on (vanishing) Carleson measures, which will be used later and have independent interests of their own.

We fix some notations which will be used through the paper. For $\delta>0$ let

$$
\begin{aligned}
\mathbb{U}_{1, \delta} & =\{z=x+i y: y>\delta\}, \\
\mathbb{U}_{2, \delta} & =\{z=x+i y: 0<y<\delta\}, \\
\mathbb{U}_{1, \delta}^{*} & =\{z=x+i y: y<-\delta\}
\end{aligned}
$$

and

$$
\mathbb{U}_{2, \delta}^{*}=\{z=x+i y:-\delta<y<0\} .
$$

Let $\lambda$ be a positive measure on the upper half plane $\mathbb{U}$. Consider

$$
M_{\delta}(\lambda)=\sup \left\{\iint_{\mathbb{U}} \frac{\eta}{|z-\bar{\zeta}|^{2}} d \lambda(z): \zeta=\xi+i \eta \in \mathbb{U}_{2, \delta}\right\},
$$


and let

$$
M(\lambda)=\lim _{\delta \rightarrow+\infty} M_{\delta}(\lambda)
$$

and

$$
M_{0}(\lambda)=\lim _{\delta \rightarrow 0+} M_{\delta}(\lambda)
$$

Then we have the following result.

Proposition 3.1. Let $\lambda$ be a positive measure on the upper half plane $\mathbb{U}$. Then $\lambda$ is a Carleson measure if and only if $M(\lambda)<+\infty$, and $N(\lambda) \asymp M(\lambda)$, while $\lambda$ is a vanishing Carleson measure if and only if $M(\lambda)<+\infty$, and $M_{0}(\lambda)=0$.

Proof. The first statement was contained (without proof) in Garnett's wellknown book [Gar]. We will write down a detailed proof, which also gives the proof of the second statement simultaneously.

First suppose that $M(\lambda)<+\infty$. For $x \in \mathbb{R}$ and $h>0$, set $\zeta=x+\frac{i}{2} h$. Noting that $|w-\bar{\zeta}| \leq 2 h$ whenever $w \in Q=Q(x, h)$, we have

$$
\lambda(Q)=\iint_{Q} d \lambda(w) \leq 4 h \iint_{Q} \frac{h}{|w-\bar{\zeta}|^{2}} d \lambda(w) \leq 4 h \iint_{\mathbb{U}} \frac{h}{|w-\bar{\zeta}|^{2}} d \lambda(w) \leq 8 h M_{\frac{h}{2}}(\lambda)
$$

which implies that

$$
\frac{\lambda(Q)}{h} \leq 8 M_{\frac{h}{2}}(\lambda)
$$

Consequently, for each $\delta>0, N_{2 \delta}(\lambda) \leq 8 M_{\delta}(\lambda)$. In particular, $N(\lambda) \leq 8 M(\lambda)$, and $N_{0}(\lambda) \leq 8 M_{0}(\lambda)$.

Conversely, suppose that $\lambda$ is a Carleson measure so that $N(\lambda)<+\infty$. Let $\zeta=\xi+i \eta \in \mathbb{U}$. For $n=0,1,2, \cdots$, set

$$
Q_{n}=Q\left(\xi, 2^{n+1} \eta\right)=\left\{z=x+i y: \xi-2^{n} \eta<x<\xi+2^{n} \eta, 0<y<2^{n+1} \eta\right\} .
$$

Then, with $Q_{-1}=\varnothing$, we have

$$
\begin{aligned}
\iint_{\mathbb{U}} \frac{\eta}{|z-\bar{\zeta}|^{2}} d \lambda(z) & =\sum_{n=0}^{+\infty} \iint_{Q_{n} \backslash Q_{n-1}} \frac{\eta}{|z-\bar{\zeta}|^{2}} d \lambda(z) \leq \sum_{n=0}^{+\infty} \iint_{Q_{n} \backslash Q_{n-1}} \frac{\eta}{\left(2^{n-1} \eta\right)^{2}} d \lambda(z) \\
& \leq \sum_{n=0}^{+\infty} \frac{2^{2-2 n}}{\eta} \iint_{Q_{n}} d \lambda(z) \leq \sum_{n=0}^{+\infty} 2^{3-n} N_{2^{n+1} \eta}(\lambda) .
\end{aligned}
$$

Consequently, for each $\delta>0$,

$$
M_{\delta}(\lambda) \leq \sum_{n=0}^{+\infty} 2^{3-n} N_{2^{n+1} \delta}(\lambda) .
$$

So

$$
M(\lambda) \leq \sum_{n=0}^{+\infty} 2^{3-n} N(\lambda)=16 N(\lambda) .
$$

Now suppose $\lambda$ is a vanishing Carleson measure so that $N(\lambda)<+\infty$, and $N_{0}(\lambda)=$ 0 . For any $\epsilon>0$, choose $n_{0}$ sufficiently large so that $2^{-n_{0}} N(\lambda)<\epsilon$. Then there exists 
some $\delta_{0}>0$ such that $N_{2^{n_{0}+1} \delta}(\lambda)<\epsilon$ whenever $\delta<\delta_{0}$. Consequently, when $\delta<\delta_{0}$, it follows from (3.4) that

$$
\begin{aligned}
M_{\delta}(\lambda) & \leq \sum_{n=0}^{n_{0}} 2^{3-n} N_{2^{n+1} \delta}(\lambda)+\sum_{n=n_{0}+1}^{+\infty} 2^{3-n} N_{2^{n+1}}(\lambda) \\
& \leq 16 N_{2^{n_{0}+1} \delta}(\lambda)+2^{3-n_{0}} N(\lambda) \leq 32 \epsilon,
\end{aligned}
$$

which implies that $M_{0}(\lambda)=0$.

Lemma 3.2. Let $s$ and $t$ be two real numbers such that $s>-1$, and $0 \leq t<s+2$. Then for $z, w, \zeta=\xi+i \eta$ in $\mathbb{U}$,

$$
\iint_{\mathbb{U}} \frac{|\zeta-\bar{\zeta}|^{s}}{|z-\bar{\zeta}|^{t}|w-\bar{\zeta}|^{2 s-t+4}} d \xi d \eta \lesssim \frac{1}{|w-\bar{z}|^{t}|w-\bar{w}|^{s-t+2}}
$$

Proof. (3.5) can be deduced from a known result of Zhao [Zh] in the unit disk case. Under the assumption that $s>-1$, and $0 \leq t<s+2$, for $Z, W, \hat{\zeta}=\hat{\xi}+i \hat{\eta}$ in $\Delta$, Zhao [Zh] showed that

$$
\iint_{\Delta} \frac{\left(1-|\hat{\zeta}|^{2}\right)^{s}}{|1-\bar{Z} \hat{\zeta}|^{t}|1-\bar{W} \hat{\zeta}|^{2 s-t+4}} d \hat{\xi} d \hat{\eta} \lesssim \frac{1}{|1-\bar{Z} W|^{t}\left(1-|W|^{2}\right)^{s-t+2}}
$$

Now we consider any Möbius transformation $\gamma: \mathbb{U} \rightarrow \Delta$. Then for any two points $\zeta_{1}$, $\zeta_{2}$ in $\mathbb{U}$, it holds that

$$
\frac{\gamma\left(\zeta_{1}\right)-\gamma\left(\zeta_{2}\right)}{1-\overline{\gamma\left(\zeta_{2}\right)} \gamma\left(\zeta_{1}\right)}=i \frac{\gamma^{\prime}\left(\zeta_{2}\right)}{\left|\gamma^{\prime}\left(\zeta_{2}\right)\right|} \frac{\zeta_{1}-\zeta_{2}}{\zeta_{1}-\bar{\zeta}_{2}}
$$

Then

$$
\frac{\left|\gamma^{\prime}\left(\zeta_{2}\right)\right|}{1-\left|\gamma\left(\zeta_{2}\right)\right|^{2}}=\frac{i}{\zeta_{2}-\bar{\zeta}_{2}}
$$

and

$$
\frac{\gamma^{\prime}\left(\zeta_{1}\right) \overline{\gamma^{\prime}\left(\zeta_{2}\right)}}{\left(1-\overline{\gamma\left(\zeta_{2}\right)} \gamma\left(\zeta_{1}\right)\right)^{2}}=-\frac{1}{\left(\zeta_{1}-\bar{\zeta}_{2}\right)^{2}}
$$

For $z, w, \zeta=\xi+i \eta$ in $\mathbb{U}$, setting $Z=\gamma(z), W=\gamma(w)$, and $\hat{\zeta}=\gamma(\zeta)$, we conclude from (3.7)-(3.9) that the left side of (3.6) is

$$
L=\frac{1}{\left|\gamma^{\prime}(z)\right|^{\frac{t}{2}}\left|\gamma^{\prime}(w)\right|^{s+2-\frac{t}{2}}} \iint_{\mathbb{U}} \frac{|\zeta-\bar{\zeta}|^{s}}{|z-\bar{\zeta}|^{t}|w-\bar{\zeta}|^{2 s-t+4}} d \xi d \eta
$$

while the right side of (3.6) is

$$
R=\frac{1}{\left|\gamma^{\prime}(z)\right|^{\frac{t}{2}}\left|\gamma^{\prime}(w)\right|^{s+2-\frac{t}{2}}} \frac{1}{|w-\bar{z}|^{t}|w-\bar{w}|^{s-t+2}} .
$$

Now (3.5) follows from $L \lesssim R$ immediately.

Proposition 3.3. Let $\lambda$ be a positive measure on the upper half plane $\mathbb{U}$. For $\alpha>0$, set

$$
\tilde{\lambda}(z)=\iint_{\mathbb{U}} \frac{y^{\alpha} v^{\alpha}}{|w-\bar{z}|^{2 \alpha+2}} d \lambda(w), \quad w=u+i v, \quad z=x+i y \in \mathbb{U} .
$$

Then $\tilde{\lambda} \in C M(\mathbb{U})$ if $\lambda \in C M(\mathbb{U})$, and $N(\tilde{\lambda}) \lesssim N(\lambda)$, while $\tilde{\lambda} \in C M_{0}(\mathbb{U})$ if $\lambda \in$ $C M_{0}(\mathbb{U})$. 
Proof. It follows from Lemma 3.2 that

$$
\begin{aligned}
\iint_{\mathbb{U}} \frac{\eta}{|z-\bar{\zeta}|^{2}} \tilde{\lambda}(z) d x d y & =\iint_{\mathbb{U}} \eta v^{\alpha} d \lambda(w) \iint_{\mathbb{U}} \frac{y^{\alpha}}{|\zeta-\bar{z}|^{2}|w-\bar{z}|^{2 \alpha+2}} \lambda(w) d u d v \\
& \lesssim \iint_{\mathbb{U}} \frac{\eta}{|w-\bar{\zeta}|^{2}} d \lambda(w)
\end{aligned}
$$

which implies that $M(\tilde{\lambda}) \lesssim M(\lambda)$, and $M_{0}(\tilde{\lambda}) \lesssim M_{0}(\lambda)$. Now Proposition 3.1 implies that $\tilde{\lambda} \in C M(\mathbb{U})$ if $\lambda \in C M(\mathbb{U})$, and $N(\tilde{\lambda}) \lesssim N(\lambda)$, while $\tilde{\lambda} \in C M_{0}(\mathbb{U})$ if $\lambda \in$ $C M_{0}(\mathbb{U})$.

Proposition 3.4. Let $\lambda$ be a positive measure on the upper half plane $\mathbb{U}$. For each $\delta>0$ set $\lambda_{\delta}=\lambda \chi_{\delta}$, where $\chi_{\delta}$ means the characteristic function on the set $\mathbb{U}_{1, \delta}$. Then $\lambda \in C M_{0}(\mathbb{U})$ if and only if $N\left(\lambda-\lambda_{\delta}\right) \rightarrow 0$ as $\delta \rightarrow 0+$.

Proof. Suppose $N\left(\lambda-\lambda_{\delta}\right) \rightarrow 0$ as $\delta \rightarrow 0+$. Then for any $\epsilon>0$ there exists some $\delta_{0}>0$ such that $N\left(\lambda-\lambda_{\delta_{0}}\right)<\epsilon$. Thus for $x \in \mathbb{R}$ and $h>0,\left(\lambda-\lambda_{\delta_{0}}\right)(Q(x, h))<\epsilon h$, which implies that

$$
\lambda(Q(x, h))=\left(\lambda-\lambda_{\delta_{0}}\right)(Q(x, h))<\epsilon h
$$

when $h<\delta_{0}$. Consequently, $\lambda \in C M_{0}(\mathbb{U})$.

Conversely, suppose $\lambda \in C M_{0}(\mathbb{U})$. Then for any $\epsilon>0$ there exists some $h_{0}>0$ such that $\lambda(Q(x, h))<\epsilon h$ whenever $x \in \mathbb{R}$ and $0<h<h_{0}$. Set $\delta_{0}=h_{0} / 2$. Then, when $0<h<h_{0}$,

$$
\left(\lambda-\lambda_{\delta_{0}}\right)(Q(x, h)) \leq \lambda(Q(x, h))<\epsilon h .
$$

Now suppose $h \geq h_{0}$. Choose an integer $n$ such that $n h_{0} \leq h \leq(n+1) h_{0}$. Then

$$
\left(\lambda-\lambda_{\delta_{0}}\right)(Q(x, h)) \leq\left(\lambda-\lambda_{\delta_{0}}\right)\left(Q\left(x,(n+1) h_{0}\right) \cap \mathbb{U}_{2, \delta_{0}}\right)<(n+1) h_{0} \epsilon \leq 2 \epsilon h .
$$

Thus $N\left(\lambda-\lambda_{\delta_{0}}\right)<2 \epsilon$, which implies that $N\left(\lambda-\lambda_{\delta}\right) \rightarrow 0$ as $\delta \rightarrow 0+$.

\section{Barycentric extension}

Douady-Earle [DE] defined in a conformally natural way a barycentric extension operator that extends a homeomorphism on the unit circle $S^{1}$ to a homeomorphism of the closed disk $\bar{\Delta}$. In this section, we will translate this operator to the setting of the real line. This operator will provide a concrete way to extend a strongly symmetric homeomorphism to an asymptotically conformal mapping on the upper half plane whose Beltrami coefficient $\mu$ induces a vanishing Carleson measure $\lambda_{\mu} \in C M_{0}(\mathbb{U})$, which implies that a strongly symmetric homeomorphism is actually symmetric.

The barycentric extension operator ex sends a sense-preserving homeomorphism $g$ of the unit circle $S^{1}$ to a quasiconformal self-mapping ex $(g)$ of the unit disk $\Delta$, which is determined by the following way: for each $Z \in \Delta, W=\operatorname{ex}(g)(Z)$ is the unique point in $\Delta$ such that

$$
G_{g}(Z, W)=\frac{1}{2 \pi} \int_{S^{1}}\left(\frac{g(\zeta)-W}{1-\bar{W} g(\zeta)}\right) \frac{1-|Z|^{2}}{|Z-\zeta|^{2}}|d \zeta|=0
$$

Although the most distinguished property of the barycentric extension operator ex is its conformally natural property (see [DE], [EMS], [EN]), we will make use of two other basic properties of ex, namely,

(1) both $\operatorname{ex}(g)$ and its inverse $\mathrm{ex}^{-1}(g)$ are real analytic; 
(2) when $g$ has a quasiconformal extension to the unit disk, $\operatorname{ex}(g)$ is quasiconformal and is even bi-Lipschitz under the Poincaré metric, that is,

$$
\frac{|d(\operatorname{ex}(g))(Z)|}{1-|\operatorname{ex}(g)(Z)|^{2}} \asymp C(g) \frac{|d Z|}{1-|Z|^{2}}, \quad Z \in \Delta .
$$

We will also need a result of $\mathrm{Cui}[\mathrm{Cu}]$ (see also [CZ]) which gives an estimate of the Beltrami coefficient of the inverse mapping $\operatorname{ex}^{-1}(g)$ : Let $\hat{\mu}$ be the Beltrami coefficient of a quasiconformal extension of $g^{-1}$, and $\hat{\nu}$ be the Beltrami coefficient of the inverse mapping $\operatorname{ex}^{-1}(g)$. Then it holds that

$$
|\hat{\nu}(W)|^{2} \leq C(g) \iint_{\Delta}|\hat{\mu}(Z)|^{2} \frac{\left(1-|W|^{2}\right)^{2}}{|1-\bar{W} Z|^{4}} d X d Y, \quad W \in \Delta .
$$

Now let $h$ be an increasing homeomorphism on the real line. Consider the Cayley transformation $\gamma(z)=(z-i) /(z+i)$ which maps $\mathbb{U}$ onto $\Delta$. Set $g=\gamma \circ h \circ \gamma^{-1}$, and define $\operatorname{ex}(h)=\gamma^{-1} \circ \operatorname{ex}(g) \circ \gamma$. We call ex $(h)$ the barycentric extension of $h$. For $\mu \in M(\mathbb{U})$, we denote by $\sigma(\mu)$ the Beltrami coefficient of the inverse mapping $\operatorname{ex}^{-1}\left(\left(\left.f^{\mu}\right|_{\mathbb{R}}\right)^{-1}\right)$. Then we have the following result.

Proposition 4.1. Let $h$ be an increasing homeomorphism on the real line. Then

(1) for each $z \in \mathbb{U}, w=\operatorname{ex}(h)(z)$ is the unique point in $\mathbb{U}$ such that

$$
H_{h}(z, w)=\frac{1}{2 \pi} \int_{\mathbb{R}}\left(\frac{h(t)-w}{h(t)-\bar{w}}\right) \frac{z-\bar{z}}{|t-\bar{z}|^{2}} d t=0 ;
$$

(2) both $\operatorname{ex}(h)$ and its inverse $\mathrm{ex}^{-1}(h)$ are real analytic;

(3) when $h$ is quasisymmetric, $\operatorname{ex}(h)$ is quasiconformal and is bi-Lipschitz under the Poincaré metric, that is,

$$
\frac{|d(\operatorname{ex}(h))(z)|}{\mid \operatorname{ex}(h)(z)-\overline{\operatorname{ex}(h)(z) \mid}} \asymp C(h) \frac{|d z|}{|z-\bar{z}|}, \quad z \in \mathbb{U} ;
$$

(4) for each $\mu \in M(\mathbb{U})$, it holds that

$$
|\sigma(\mu)(w)|^{2} \leq C\left(\|\mu\|_{\infty}\right) \iint_{\mathbb{U}}|\mu(z)|^{2} \frac{|w-\bar{w}|^{2}}{|z-\bar{w}|^{4}} d x d y, \quad w \in \mathbb{U} .
$$

Proof. (2) and (3) are obviously true. For $z, w$ in $\mathbb{U}$, a direct computation by (3.7)-(3.9) yields that

$$
G_{g}(\gamma(z), \gamma(w))=\frac{\gamma^{\prime}(w)}{\left|\gamma^{\prime}(w)\right|} H_{h}(z, w), \quad g=\gamma \circ h \circ \gamma^{-1} .
$$

Thus, (1) follows from

$$
w=\operatorname{ex}(h)(z) \Leftrightarrow \gamma(w)=\operatorname{ex}(g) \circ \gamma(z) \Leftrightarrow G_{g}(\gamma(z), \gamma(w))=0 \Leftrightarrow H_{h}(z, w)=0 .
$$

To prove (4), let $\mu \in M(\mathbb{U})$, and $h=\left(\left.f^{\mu}\right|_{\mathbb{R}}\right)^{-1}$. Set $g=\gamma \circ h \circ \gamma^{-1}$ as above. Then $\gamma \circ f^{\mu} \circ \gamma^{-1}$ is a quasiconformal extension of $g^{-1}$ whose Beltrami coefficient $\hat{\mu}$ is $\left(\mu \circ \gamma^{-1}\right) \overline{\left(\gamma^{-1}\right)^{\prime}} /\left|\left(\gamma^{-1}\right)^{\prime}\right|$. On the other hand, since $\operatorname{ex}(h)=\gamma^{-1} \circ \operatorname{ex}(g) \circ \gamma$, the Beltrami coefficient $\hat{\nu}$ of $\operatorname{ex}^{-1}(g)$ is $\left(\sigma(\mu) \circ \gamma^{-1}\right) \overline{\left(\gamma^{-1}\right)^{\prime}} /\left|\left(\gamma^{-1}\right)^{\prime}\right|$. Now for $w \in \mathbb{U}$, setting 
$W=\gamma(w) \in \Delta$ in (4.3) we obtain by (3.8) and (3.9) that

$$
\begin{aligned}
|\sigma(\mu)(w)|^{2} & \leq C\left(\|\mu\|_{\infty}\right) \iint_{\Delta}|\hat{\mu}(Z)|^{2} \frac{\left(1-|\gamma(w)|^{2}\right)^{2}}{|1-\overline{\gamma(w)} Z|^{4}} d X d Y \\
& =C\left(\|\mu\|_{\infty}\right) \iint_{\mathbb{U}}|\mu(z)|^{2}\left|\gamma^{\prime}(z)\right|^{2} \frac{\left(1-|\gamma(w)|^{2}\right)^{2}}{|1-\overline{\gamma(w)} \gamma(z)|^{4}} d x d y \\
& =C\left(\|\mu\|_{\infty}\right) \iint_{\mathbb{U}}|\mu(z)|^{2} \frac{|w-\bar{w}|^{2}}{|z-\bar{w}|^{4}} d x d y
\end{aligned}
$$

This completes the proof.

Remark 4.2. By means of Lemma 3.3 in our paper [HWS], we conclude by (4.6) that $\sigma(\mu) \in M_{0}(\mathbb{U})$ if $\mu \in M_{0}(\mathbb{U})$, that is, $\mathrm{ex}^{-1}\left(h^{-1}\right)$ is asymptotically conformal when $h$ is a symmetric homeomorphism on the real line. This gives a somewhat concrete asymptotically conformal extension for a symmetric homeomorphism. As stated in section 1, the Beurling-Ahlfors extension of $h$ is known to be asymptotically conformal when $h$ is symmetric (see [GS], [Mat]).

Now we prove the main result in this section.

Theorem 4.3. For each $\mu \in M(\mathbb{U}), \sigma(\mu) \in \mathcal{M}(\mathbb{U})$ if $\mu \in \mathcal{M}(\mathbb{U})$, and $\|\sigma(\mu)\|_{c} \lesssim$ $\|\mu\|_{c}$, while $\sigma(\mu) \in \mathcal{M}_{0}(\mathbb{U}) \cap M_{0}(\mathbb{U})$ if $\mu \in \mathcal{M}_{0}(\mathbb{U})$.

Proof. Except the statement that $\sigma(\mu) \in M_{0}(\mathbb{U})$, the result follows from Propositions $3.3(\alpha=1)$ and 4.1. To prove $\sigma(\mu) \in M_{0}(\mathbb{U})$ if $\mu \in \mathcal{M}_{0}(\mathbb{U})$, we follow the same discussion used to prove Proposition 3.1.

Let $\zeta=\xi+i \eta \in \mathbb{U}$. For $n=0,1,2, \cdots$, set as before that

$$
Q_{n}=Q\left(\xi, 2^{n+1} \eta\right)=\left\{z=x+i y: \xi-2^{n} \eta<x<\xi+2^{n} \eta, 0<y<2^{n+1} \eta\right\} .
$$

Then, with $Q_{-1}=\varnothing$, we have

$$
\begin{aligned}
|\sigma(\mu)(\zeta)|^{2} & \leq C\left(\|\mu\|_{\infty}\right) \iint_{\mathbb{U}}|\mu(z)|^{2} \frac{\eta^{2}}{|z-\bar{\zeta}|^{4}} d x d y \\
& =C\left(\|\mu\|_{\infty}\right) \sum_{n=0}^{+\infty} \iint_{Q_{n} \backslash Q_{n-1}} \frac{y \eta^{2}}{|z-\bar{\zeta}|^{4}} \lambda_{\mu}(z) d x d y \\
& \leq C\left(\|\mu\|_{\infty}\right) \sum_{n=0}^{+\infty} \iint_{Q_{n} \backslash Q_{n-1}} \frac{2^{n+1} \eta^{3}}{\left(2^{n-1} \eta\right)^{4}} \lambda_{\mu}(z) d x d y \\
& \leq C\left(\|\mu\|_{\infty}\right) \sum_{n=0}^{+\infty} \frac{2^{5-3 n}}{\eta} \iint_{Q_{n}} \lambda_{\mu}(z) d x d y \\
& \leq C\left(\|\mu\|_{\infty}\right) \sum_{n=0}^{+\infty} 2^{5-3 n} N_{2^{n+1} \eta}\left(\lambda_{\mu}\right) .
\end{aligned}
$$

Consequently, we conclude by the same reasoning as in the proof of Proposition 3.1 that $\sigma(\mu)(\xi+i \eta) \rightarrow 0$ uniformly for $\xi \in \mathbb{R}$ as $\eta \rightarrow 0+$, that is, $\sigma(\mu) \in M_{0}(\mathbb{U})$.

Remark 4.4. Proposition 4.1 and Theorem 4.3 will play an essential role in the next section where we prove the existence of local holomorphic sections of the Bers projection $\mathcal{S}: \mathcal{M}_{0}(\mathbb{U}) \rightarrow \mathcal{B}_{0}\left(\mathbb{U}^{*}\right)$. Theorem 4.3 also implies that a strongly symmetric homeomorphism must be symmetric, as we have promised in section 1 . This fact will also be used in the proof of Theorems 7.6 and 8.2. 


\section{Proof of Theorem 2.1}

In this section, we give the proof of Theorem 2.1, the first main result of this paper. We first note

Proposition 5.1. For each $\mu \in M(\mathbb{U})$, it holds that $\mathcal{S}(\mu) \in \mathcal{B}\left(\mathbb{U}^{*}\right)$ if $\mu \in \mathcal{M}(\mathbb{U})$, while $\mathcal{S}(\mu) \in \mathcal{B}_{0}\left(\mathbb{U}^{*}\right)$ if $\mu \in \mathcal{M}_{0}(\mathbb{U})$.

Proof. In [HWS] we established the following integral representation of the Schwarzian derivative $\mathcal{S}(\mu)$ :

$$
\mathcal{S}(\mu)(z)=-\frac{6}{\pi} f_{\mu}^{\prime}(z) \iint_{\mathbb{U}} \frac{\bar{\partial} f_{\mu}(\zeta)}{(\zeta-z)^{2}\left(f_{\mu}(\zeta)-f_{\mu}(z)\right)^{2}} d \xi d \eta, \quad z \in \mathbb{U}^{*},
$$

from which we deduced the following estimate:

$$
|\mathcal{S}(\mu)(z)|^{2} \leq \frac{C\left(\|\mu\|_{\infty}\right)}{y^{2}} \iint_{\mathbb{U}} \frac{|\mu(\zeta)|^{2}}{|\zeta-z|^{4}} d \xi d \eta, \quad z=x+i y \in \mathbb{U}^{*}
$$

Now the result follows directly from Proposition $3.3(\alpha=1)$.

Remark 5.2. The first statement of Proposition 5.1 (in the unit disk case) was already proved by Astala-Zinsmeister [AZ]. The second statement implies the inclusion relation $\beta\left(T_{v}\right) \subset \beta(T) \cap \mathcal{B}_{0}\left(\mathbb{U}^{*}\right)$. It is not clear whether the converse is true.

Proof of Theorem 2.1. Proposition 5.1 says that $\mathcal{S}$ maps $\mathcal{M}_{0}(\mathbb{U})$ into $\mathcal{B}_{0}\left(\mathbb{U}^{*}\right)$. This also implies that $\mathcal{S}: \mathcal{M}_{0}(\mathbb{U}) \rightarrow \mathcal{B}_{0}\left(\mathbb{U}^{*}\right)$ is holomorphic since $\mathcal{S}: \mathcal{M}(\mathbb{U}) \rightarrow \mathcal{B}\left(\mathbb{U}^{*}\right)$ is holomorphic. It remains to show that $\mathcal{S}: \mathcal{M}_{0}(\mathbb{U}) \rightarrow \mathcal{B}_{0}\left(\mathbb{U}^{*}\right)$ has local holomorphic sections. We write down the standard proof due to Ahlfors [Ah].

Let $\phi=\mathcal{S}(\mu), \mu \in \mathcal{M}_{0}(\mathbb{U})$, be given. Without loss of generality, we may assume by Theorem 4.3 that $\mu=\sigma(\mu)$, that is, $f^{\mu}$ is the inverse of the barycentric extension of $\left(\left.f^{\mu}\right|_{\mathbb{R}}\right)^{-1}$. Set $f=f_{\mu}, D=f(\mathbb{U}), D^{*}=f\left(\mathbb{U}^{*}\right)$, and $r=f \circ \overline{f^{-1}}$. Ahlfors [Ah] showed that $r: D \rightarrow D^{*}$ is a quasiconformal reflection and there exists a constant $C_{1}=C_{1}\left(\|\mu\|_{\infty}\right)$ such that

$$
\frac{1}{C_{1}} \leq|r(z)-z|^{2} \lambda_{D^{*}}^{2}(r(z))|\bar{\partial} r(z)| \leq C_{1}, \quad z \in D .
$$

It should be pointed out that, to have the estimate (5.3), $f^{\mu}$ needs to be a biLipschitz diffeomorphism under the Poincaré metric $|d z| / y$. In the original paper [Ah], Ahlfors used the Beurling-Ahlfors [BA] extension operator, namely, $f^{\mu}$ is the Beurling-Ahlfors extension of $\left.f^{\mu}\right|_{\mathbb{R}}$, which also appeared in [GS] and our recent paper [HWS]. However, in order to prove the existence of local holomorphic sections of the Bers projection $\mathcal{S}$ restricted to some subspaces, barycentric extension operator has to be used (see [EN], [SW], [TT], [TS]), which means that $f^{\mu}$ is the barycentric extension of $\left.f^{\mu}\right|_{\mathbb{R}}$. Here we use the inverse barycentric extension operator, namely, $f^{\mu}$ is the inverse of the barycentric extension of $\left(f^{\mu} \mid \mathbb{R}\right)^{-1}$, which satisfies the required properties by Proposition 4.1 and Theorem 4.3 (see Remark 4.4). Actually, it is not clear whether the Beltrami coefficient of the Beurling-Ahlfors extension or the barycentric extension of $\left.f^{\mu}\right|_{\mathbb{R}}$ belongs to $\mathcal{M}_{0}(\mathbb{U})$ when $u \in \mathcal{M}_{0}(\mathbb{U})$.

Consider $\mathcal{B}_{\epsilon}(\phi)=\left\{\psi \in \mathcal{B}_{0}\left(\mathbb{U}^{*}\right):\|\psi-\phi\|_{\mathcal{B}}<\epsilon\right\}$ for $\epsilon>0$. Then for each $\psi \in \mathcal{B}_{\epsilon}(\phi)$ there exists a unique locally univalent function $f_{\psi}$ in $\mathbb{U}^{*}$ which fixes the points 0,1 , $\infty$ such that $S_{f_{\psi}}=\psi$. Set $g_{\psi}=f_{\psi} \circ f^{-1}$. Then $S_{g_{\psi}} \circ f\left(f^{\prime}\right)^{2}=\psi-\phi$, and

$$
\lambda_{D^{*}}^{-2}(f(z))\left|S_{g_{\psi}}(f(z))\right|=|z-\bar{z}|^{2}|\psi(z)-\phi(z)|, \quad z \in \mathbb{U}^{*} .
$$


When $\epsilon$ is small, following Ahlfors [Ah] we proved in [SW] that $g_{\psi}$ is univalent and can be extended to a quasiconformal mapping in the whole plane whose complex dilatation $\mu_{\psi}$ has the form

$$
\mu_{\psi}(z)=\frac{S_{g_{\psi}}(r(z))(r(z)-z)^{2} \bar{\partial} r(z)}{2+S_{g_{\psi}}(r(z))(r(z)-z)^{2} \partial r(z)}, \quad z \in D .
$$

By (5.3) we have for some constant $C_{2}=C_{2}\left(\|\mu\|_{\infty}\right)$ that

$$
\left|\mu_{\psi}(z)\right| \leq C_{2}\left|S_{g_{\psi}}(r(z))\right| \lambda_{D^{*}}^{-2}(r(z)), \quad z \in D .
$$

Consequently, $f_{\psi}=g_{\psi} \circ f$ is univalent in $\mathbb{U}^{*}$ and has a quasiconformal extension to the whole plane whose complex dilatation $\nu_{\psi}$ is

$$
\nu_{\psi}=\frac{\mu+\left(\mu_{\psi} \circ f\right) \tau}{1+\bar{\mu}\left(\mu_{\psi} \circ f\right) \tau}, \quad \tau=\frac{\overline{\partial f}}{\partial f} .
$$

We proved in $[\mathrm{SW}]$ that $\nu_{\psi} \in \mathcal{M}(\mathbb{U})$ depends holomorphically on $\psi$. Now, it follows from (5.6) that

$$
\begin{aligned}
\left|\mu_{\psi}(f(z))\right| & \leq C_{2}\left|S_{g_{\psi}}(r(f(z)))\right| \lambda_{D^{*}}^{-2}(r(f(z)))=C_{2}\left|S_{g_{\psi}}(f(\bar{z}))\right| \lambda_{D^{*}}^{-2}(f(\bar{z})) \\
& =C_{2}|\psi(\bar{z})-\phi(\bar{z})||z-\bar{z}|^{2}
\end{aligned}
$$

which implies that $\mu_{\psi} \circ f \in \mathcal{M}_{0}(\mathbb{U})$, and we conclude by (5.7) that $\nu_{\psi} \in \mathcal{M}_{0}(\mathbb{U})$. Since $\mathcal{S}\left(\nu_{\psi}\right)=\psi$, we conclude that $\nu: \mathcal{B}_{\epsilon}(\phi) \rightarrow \mathcal{M}_{0}(\mathbb{U})$ is a local holomorphic section to $\mathcal{S}: \mathcal{M}_{0}(\mathbb{U}) \rightarrow \mathcal{B}_{0}\left(\mathbb{U}^{*}\right)$. This completes the proof of Theorem 2.1.

\section{Pre-logarithmic derivative model of $T_{0}$}

The universal Teichmüller space has several models, one of which is the prelogarithmic derivative model (see $[\mathrm{AG}],[\mathrm{Zu}]$ ). In the unit disk case, the pre-logarithmic derivative models of the universal Teichmüller space and its various subspaces were much investigated in recent years (see [AZ], [Sh], [SW], [TT] for more details). Unlike the Schwarzian derivative model (discussed in section 2), the logarithmic derivative is not invariant under a Möbius transformation. In this section, we will deal with the pre-logarithmic derivative model of the symmetric Teichmüller space in the half plane case. The results will be used in the next section to deal with the VMO Teichmüller space.

Let $\mathbb{B}\left(\mathbb{U}^{*}\right)$ denote the Bloch space of functions $\phi$ holomorphic in $\mathbb{U}^{*}$ with seminorm

$$
\|\phi\|_{\mathbb{B}} \doteq \sup \left\{\left|\phi^{\prime}(z) \| y\right|: z=x+i y \in \mathbb{U}^{*}\right\}
$$

and $\mathbb{B}_{0}\left(\mathbb{U}^{*}\right)$ the closed subspace of $\mathbb{B}\left(\mathbb{U}^{*}\right)$ which consists of those functions $\phi$ such that $y \phi^{\prime}(x+i y) \rightarrow 0$ uniformly for $x \in \mathbb{R}$ as $y \rightarrow 0-$. Koebe distortion theorem implies that $\left.\log f_{\mu}^{\prime}\right|_{\mathbb{U}^{*}} \in \mathbb{B}\left(\mathbb{U}^{*}\right)$ for $\mu \in M(\mathbb{U})$ (see Proposition 6.1 below). Furthermore, the map $L$ induced by the correspondence $\left.\mu \mapsto \log f_{\mu}^{\prime}\right|_{\mathbb{U}^{*}}$ is a continuous map from $M(\mathbb{U})$ into $\mathbb{B}\left(\mathbb{U}^{*}\right)$ (see $[$ Le] $)$. Actually, $L: M(\mathbb{U}) \rightarrow \mathbb{B}\left(\mathbb{U}^{*}\right)$ is even holomorphic (see [Ha]).

Proposition 6.1. Let $f$ be a conformal mapping in the upper half plane. Then

$$
\left|N_{f}(z)\right| y \leq 3, \quad z=x+i y \in \mathbb{U} .
$$

Furthermore, equality (6.2) holds at $z \in \mathbb{U}$ if and only if

$$
f(\zeta)=f(z)+f^{\prime}(z)(z-\bar{z}) \frac{(\zeta-z)(\zeta-\bar{z})}{(2 \zeta-z-\bar{z})^{2}} .
$$


Proof. Proposition 6.1 should be a known result. For completeness and for later use, we write down the proof here. For each $z=x+i y \in \mathbb{U}$, consider

$$
\gamma(w)=\frac{\bar{z} w-z}{w-1}, \quad w \in \Delta,
$$

which maps the unit disk $\Delta$ onto the upper half plane $\mathbb{U}$ with $\gamma(0)=z, \gamma^{\prime}(0)=z-\bar{z}$, and $\gamma^{\prime \prime}(0)=2(z-\bar{z})$. Set

$$
g(w)=\frac{f(\gamma(w))-f(z)}{(z-\bar{z}) f^{\prime}(z)} .
$$

Then $g$ is conformal in the unit disk $\Delta$ with $g(0)=0, g^{\prime}(0)=1$, and

$$
g^{\prime \prime}(0)=N_{f}(z)(z-\bar{z})+2 .
$$

By the well-known result of Bieberbach (see $[\mathrm{Po}]$ ), we have $\left|g^{\prime \prime}(0)\right| \leq 4$, from which we obtain (6.2).

Now if equality (6.2) holds we must have $N_{f}(z)(z-\bar{z})=-6$, or equivalently, $g^{\prime \prime}(0)=-4$. On the other hand, Bieberbach's result also implies that $g(w)$ is a rotation of the Koebe function, that is,

$$
g(w)=\frac{w}{(1+w)^{2}} .
$$

Then we obtain from (6.4) that

$$
f(\zeta)=f(z)+f^{\prime}(z)(z-\bar{z}) \frac{\gamma^{-1}(\zeta)}{\left(1+\gamma^{-1}(\zeta)\right)^{2}}=f(z)+f^{\prime}(z)(z-\bar{z}) \frac{(\zeta-z)(\zeta-\bar{z})}{(2 \zeta-z-\bar{z})^{2}}
$$

as desired.

Now we show

Theorem 6.2. Given $\mu \in M(\mathbb{U})$, the following statements are all equivalent:

(1) There exists some $\nu \in M_{0}(\mathbb{U})$ such that $[\nu]=[\mu]$;

(2) $L(\mu) \in \mathbb{B}_{0}\left(\mathbb{U}^{*}\right)$

(3) $\mathcal{S}(\mu) \in B_{0}\left(\mathbb{U}^{*}\right)$

(4) $h=\left.f^{\mu}\right|_{\mathbb{R}}$ is symmetric.

Proof. As stated in section $1,(1) \Leftrightarrow(4)$ is known (see [GS], [Mat]). Now $(1) \Rightarrow(3)$ was proved in our previous paper [HWS], while $(3) \Rightarrow(1)$ was proved recently by Wei-Matsuzaki [WM1]. We will show that $(1) \Rightarrow(2) \Rightarrow(3)$ by means of some reasoning from our paper [STW].

To show $(1) \Rightarrow(2)$, we use the continuity of the map $L: M(\mathbb{U}) \rightarrow \mathbb{B}\left(\mathbb{U}^{*}\right)$. Let $\mu \in M_{0}(\mathbb{U})$ be given. For $\delta>0$, set $\mu_{\delta}=\mu \chi_{\delta}$, where $\chi_{\delta}$ is the characteristic function on the set $\mathbb{U}_{1, \delta}$. Then $\left\|\mu_{\delta}-\mu\right\|_{\infty} \rightarrow 0$ as $\delta \rightarrow 0+$. By the continuity of $L: M(\mathbb{U}) \rightarrow \mathbb{B}\left(\mathbb{U}^{*}\right)$ we obtain $\left\|L\left(\mu_{\delta}\right)-L(\mu)\right\|_{\mathbb{B}} \rightarrow 0$ as $\delta \rightarrow 0+$. On the other hand, since $f_{\mu_{\delta}}$ is conformal outside $\mathbb{U}_{1, \delta}$, it is obvious that $L\left(\mu_{\delta}\right) \in \mathbb{B}_{0}\left(\mathbb{U}^{*}\right)$. In fact, set $f_{\delta}(z)=f_{\mu_{\delta}}(z+i \delta)$. Then $f_{\delta}$ is univalent in $\mathbb{U}^{*}$, which implies by Proposition 6.1 that $\left|N_{f_{\delta}}(z)\right||y| \leq 3$ for $z=x+i y \in \mathbb{U}^{*}$. Since $N_{f_{\delta}}(z)=N_{f_{\mu_{\delta}}}(z+i \delta)$, we obtain

$$
\left|N_{f_{\mu_{\delta}}}(z)\right||y-\delta| \leq 3, z=x+i y \in \mathbb{U}^{*}
$$

which implies that $L\left(\mu_{\delta}\right) \in \mathbb{B}_{0}\left(\mathbb{U}^{*}\right)$ as desired. Since $\mathbb{B}_{0}\left(\mathbb{U}^{*}\right)$ is closed in $\mathbb{B}\left(\mathbb{U}^{*}\right)$, we conclude that $L(\mu) \in \mathbb{B}_{0}\left(\mathbb{U}^{*}\right)$.

Noting that

$$
\mathcal{S}(\mu)=L^{\prime \prime}(\mu)-\frac{1}{2}\left(L^{\prime}(\mu)\right)^{2},
$$


we conclude that $(2) \Rightarrow(3)$ follows immediately from the following lemma. Here and in what follows, we use $L^{\prime}(\mu)$ and $L^{\prime \prime}(\mu)$ respectively to denote the first and second order derivatives of $L(\mu)$.

Lemma 6.3. Let $\phi$ be a holomorphic function on the lower half plane $\mathbb{U}^{*}$ such that $\lim _{y \rightarrow-\infty} \phi^{\prime}(x+i y)=0$ uniformly for $x \in \mathbb{R}$. Then $\phi \in \mathbb{B}\left(\mathbb{U}^{*}\right)$ if and only if $\phi^{\prime \prime} \in B\left(\mathbb{U}^{*}\right)$, and $\|\phi\|_{\mathbb{B}} \asymp\left\|\phi^{\prime \prime}\right\|_{B\left(\mathbb{U}^{*}\right)}$, while $\phi \in \mathbb{B}_{0}\left(\mathbb{U}^{*}\right)$ if and only if $\phi^{\prime \prime} \in B_{0}\left(\mathbb{U}^{*}\right)$.

Proof. Suppose $\phi \in \mathbb{B}\left(\mathbb{U}^{*}\right)$. For $z=x+i y \in \mathbb{U}^{*}$, it follows from the Cauchy integral formula that

$$
\left|\phi^{\prime \prime}(z)\right|=\left|\frac{1}{2 \pi i} \int_{|\zeta-z|=|y| / 4} \frac{\phi^{\prime}(\zeta)}{(\zeta-z)^{2}} d \zeta\right| \leq 4 /|y| \sup _{|\zeta-z|<|y| / 4}\left|\phi^{\prime}(\zeta)\right| .
$$

Noting that the function $\left|\phi^{\prime}\right|^{2}$ is subharmonic in $\mathbb{U}^{*}$, we have

$$
\left|\phi^{\prime}(\zeta)\right|^{2} \leq \frac{16}{\pi y^{2}} \iint_{|w-\zeta|<|y| / 4}\left|\phi^{\prime}(w)\right|^{2} d u d v, \quad|\zeta-z|<|y| / 4 .
$$

By (6.7) we obtain

$$
y^{4}\left|\phi^{\prime \prime}(z)\right|^{2} \leq \frac{256}{\pi} \int_{2 y<v<y / 2} \int_{|u-x|<|y|}\left|\phi^{\prime}(w)\right|^{2} d u d v .
$$

Then

$$
y^{4}\left|\phi^{\prime \prime}(z)\right|^{2} \leq \frac{256\|\phi\|_{\mathbb{B}}^{2}}{\pi} \int_{2 y<v<y / 2} \int_{|u-x|<|y|}|v|^{-2} d u d v=\frac{768\|\phi\|_{\mathbb{B}}^{2}}{\pi},
$$

which implies that $\phi^{\prime \prime} \in B\left(\mathbb{U}^{*}\right)$ with $\left\|\phi^{\prime \prime}\right\|_{B\left(\mathbb{U}^{*}\right)} \lesssim\|\phi\|_{\mathbb{B}}$. If $\phi \in \mathbb{B}_{0}\left(\mathbb{U}^{*}\right)$, then for every $\epsilon>0$, there is some $\delta_{0}>0$ such that

$$
|v|\left|\phi^{\prime}(w)\right|<\epsilon, \quad w=u+i v \in \mathbb{U}_{2, \delta_{0}}^{*} .
$$

Now we let $z=x+i y \in \mathbb{U}_{2, \delta_{0} / 2}^{*}$. It is easy to see that $w=u+i v \in \mathbb{U}_{2, \delta_{0}}^{*}$ whenever $2 y<v<y / 2$ and $|u-x|<|y|$. Thus it follows from (6.8) and (6.6) that

$$
y^{4}\left|\phi^{\prime \prime}(z)\right|^{2} \leq \frac{256 \epsilon^{2}}{\pi} \int_{2 y<v<y / 2} \int_{|u-x|<|y|} v^{-2} d u d v=\frac{768 \epsilon^{2}}{\pi},
$$

which implies that $\phi^{\prime \prime} \in B_{0}\left(\mathbb{U}^{*}\right)$.

Conversely, suppose $\phi^{\prime \prime} \in B\left(\mathbb{U}^{*}\right)$. Since $\lim _{y \rightarrow-\infty} \phi^{\prime}(x+i y)=0$ uniformly for $x \in \mathbb{R}$, it holds that

$$
\phi^{\prime}(x+i y)=i \int_{-\infty}^{y} \phi^{\prime \prime}(x+i v) d v
$$

which implies that

$$
\left|\phi^{\prime}(x+i y)\right| \leq \int_{-\infty}^{y}\left|\phi^{\prime \prime}(x+i v)\right| d v
$$

Thus

$$
\left|\phi^{\prime}(x+i y)\right| \leq \int_{-\infty}^{y}\left|\phi^{\prime \prime}(x+i v)\right| d v \leq\left\|\phi^{\prime \prime}\right\|_{B\left(\mathbb{U}^{*}\right)} \int_{-\infty}^{y}|v|^{-2} d v=\frac{\left\|\phi^{\prime \prime}\right\|_{B\left(\mathbb{U}^{*}\right)}}{|y|},
$$

which implies $\phi \in \mathbb{B}\left(\mathbb{U}^{*}\right)$ with $\|\phi\|_{\mathbb{B}} \leq\left\|\phi^{\prime \prime}\right\|_{B\left(\mathbb{U}^{*}\right)}$. If $\phi^{\prime \prime} \in B_{0}\left(\mathbb{U}^{*}\right)$, then for every $\epsilon>0$, there is some $\delta_{0}>0$ such that

$$
|v|^{2}\left|\phi^{\prime \prime}(w)\right|<\epsilon, \quad w=u+i v \in \mathbb{U}_{2, \delta_{0}}^{*} .
$$


Then, when $z=x+i y \in \mathbb{U}_{2, \delta_{0}}^{*}$,

$$
\begin{aligned}
\left|\phi^{\prime}(x+i y)\right| & \leq \int_{-\infty}^{y}\left|\phi^{\prime \prime}(x+i v)\right| d v \\
& =\int_{-\infty}^{-\delta_{0}}\left|\phi^{\prime \prime}(x+i v)\right| d v+\int_{-\delta_{0}}^{y}\left|\phi^{\prime \prime}(x+i v)\right| d v \\
& \leq\left\|\phi^{\prime \prime}\right\|_{B\left(\mathbb{U}^{*}\right)} \int_{-\infty}^{-\delta_{0}}|v|^{-2} d v+\epsilon \int_{-\delta_{0}}^{y}|v|^{-2} d v \\
& =\frac{\left\|\phi^{\prime \prime}\right\|_{B\left(\mathbb{U}^{*}\right)}}{\delta_{0}}-\epsilon\left(\frac{1}{\delta_{0}}+\frac{1}{y}\right) .
\end{aligned}
$$

Thus,

$$
\left|y \| \phi^{\prime}(x+i y)\right| \leq \frac{\left\|\phi^{\prime \prime}\right\|_{B\left(\mathbb{U}^{*}\right)}}{\delta_{0}}|y|+\epsilon,
$$

which implies $|y|\left|\phi^{\prime}(x+i y)\right| \rightarrow 0$ uniformly for $x \in \mathbb{R}$ as $y \rightarrow 0-$, that is, $\phi \in$ $\mathbb{B}_{0}\left(\mathbb{U}^{*}\right)$.

\section{Pre-logarithmic derivative model of $T_{v}$}

We begin with the following basic result.

Lemma 7.1. Let $\phi$ be analytic in $\mathbb{U}^{*}, n \in \mathbb{N}, \alpha>0$. Set $\lambda(z)=|\phi(z)|^{n}|y|^{\alpha}$ for $z=x+i y \in \mathbb{U}^{*}$. Then the following statements hold:

(1) If $\lambda \in C M\left(\mathbb{U}^{*}\right)$, then $\sup _{z \in \mathbb{U}^{*}}|\phi(z)|^{n}|y|^{\alpha+1}<C(\alpha) N(\lambda)$;

(2) If $\lambda \in C M_{0}\left(\mathbb{U}^{*}\right)$, then $\lim _{y \rightarrow 0}|\phi(z)|^{n}|y|^{\alpha+1}=0$.

Proof. For $x_{0} \in \mathbb{R}$ and $h>0$, let

$$
Q^{*}\left(x_{0}, h\right)=\left\{z=x+i y: x_{0}-h / 2<x<x_{0}+h / 2,-h<y<0\right\} .
$$

Denote by $D(\zeta, r)$ the ball of center $\zeta$ with radius $r$. For any $z \in \mathbb{U}^{*}$, it is easy to see that $D(z,|y| / 2) \subset Q^{*}(x, 3|y| / 2)$. Thus,

$$
\iint_{D(z,|y| / 2)}|\phi(\zeta)|^{n}|\eta|^{\alpha} d \xi d \eta \leq \lambda\left(Q^{*}(x, 3|y| / 2)\right) .
$$

On the other hand, for $\zeta=\xi+i \eta \in D(z,|y| / 2),|\eta| \geq|y| / 2$, so

$$
\iint_{D(z,|y| / 2)}|\phi(\zeta)|^{n}|\eta|^{\alpha} d \xi d \eta \geq \frac{y^{\alpha}}{2^{\alpha}} \iint_{D(z,|y| / 2)}|\phi(\zeta)|^{n} d \xi d \eta \geq \pi \frac{|y|^{\alpha+2}}{2^{\alpha+2}}|\phi(z)|^{n} .
$$

Consequently,

$$
|\phi(z)|^{n}|y|^{\alpha+1} \leq \pi 2^{\alpha+2} \frac{\lambda\left(Q^{*}(x, 3|y| / 2)\right)}{|y|},
$$

from which we obtain the required results immediately.

Now let BMOA $\left(\mathbb{U}^{*}\right)$ denote the space of all functions $\phi$ which are holomorphic in $\mathbb{U}^{*}$ and of bounded mean oscillation (see [FS], [Gar]), or equivalently, $\phi$ induces a Carleson measure $\kappa_{\phi}$ by $\kappa_{\phi}(z)=\left|\phi^{\prime}(z)\right|^{2}|y| d x d y \in C M\left(\mathbb{U}^{*}\right), z=x+i y \in \mathbb{U}^{*}$. The semi-norm on $\operatorname{BMOA}\left(\mathbb{U}^{*}\right)$ is

$$
\|\phi\|_{\mathrm{BMOA}}=\sqrt{N\left(\kappa_{\phi}\right)}
$$


Lemma 7.1 implies that $\mathrm{BMOA}\left(\mathbb{U}^{*}\right) \subset \mathbb{B}\left(\mathbb{U}^{*}\right)$, and the inclusion map is continuous. We also denote by $\operatorname{VMOA}\left(\mathbb{U}^{*}\right)$ the closed subspace of $\operatorname{BMOA}\left(\mathbb{U}^{*}\right)$ which consists of all holomorphic functions $\phi$ of vanishing mean oscillation (see [FS], [Gar], [Sa]), or equivalently, $\phi$ induces a vanishing Carleson measure $\kappa_{\phi} \in C M_{0}\left(\mathbb{U}^{*}\right)$. Then $\operatorname{VMOA}\left(\mathbb{U}^{*}\right) \subset \mathbb{B}_{0}\left(\mathbb{U}^{*}\right)$ by Lemma 7.1 again.

We recall the following known result on the BMO-Teichmüller space (see [AZ], [BJ], [FKS], [Ma], [Se2] and Remark 1.1).

Theorem 7.2. Let $\mu \in M(\mathbb{U})$ be given. Then the following statements are equivalent:

(1) There exists some $\nu \in \mathcal{M}(\mathbb{U})$ such that $[\nu]=[\mu]$;

(2) $L(\mu) \in \operatorname{BMOA}\left(\mathbb{U}^{*}\right)$

(3) $\mathcal{S}(\mu) \in \mathcal{B}\left(\mathbb{U}^{*}\right)$

(4) $h=\left.f^{\mu}\right|_{\mathbb{R}}$ is strongly quasisymmetric.

We now consider how to obtain a similar result on the VMO-Teichmüller space. We need the following result.

Lemma 7.3. Let $\phi$ be a holomorphic function on the lower half plane $\mathbb{U}^{*}$ such that $\lim _{y \rightarrow-\infty} \phi^{\prime}(x+i y)=0$ uniformly for $x \in \mathbb{R}$. Then $\phi \in \operatorname{BMOA}\left(\mathbb{U}^{*}\right)$ if and only if $\phi^{\prime \prime} \in \mathcal{B}\left(\mathbb{U}^{*}\right)$, and $\left\|\phi^{\prime \prime}\right\|_{\mathcal{B}} \asymp\|\phi\|_{\mathrm{BMOA}}$, while $\phi \in \operatorname{VMOA}\left(\mathbb{U}^{*}\right)$ if and only if $\phi^{\prime \prime} \in \mathcal{B}_{0}\left(\mathbb{U}^{*}\right)$.

Proof. Suppose $\phi \in \operatorname{BMOA}\left(\mathbb{U}^{*}\right)$. For $z=x+i y \in \mathbb{U}^{*}$, it follows from (6.8) that

$$
\begin{aligned}
y^{4}\left|\phi^{\prime \prime}(z)\right|^{2} & \lesssim \int_{x+y}^{x-y} d u \int_{2 y}^{\frac{y}{2}} d v\left|\phi^{\prime}(u+i v)\right|^{2} \\
& =y^{2} \int_{-1}^{1} d \xi \int_{-2}^{-\frac{1}{2}} d \eta\left|\phi^{\prime}(x-y \xi-i y \eta)\right|^{2} .
\end{aligned}
$$

Then

$$
\begin{aligned}
\iint_{Q^{*}\left(x_{0}, h\right)} \lambda_{\phi^{\prime \prime}}(z) d x d y & \lesssim \int_{x_{0}-h}^{x_{0}+h} d x \int_{-h}^{0} d y \int_{-1}^{1} d \xi \int_{-2}^{-\frac{1}{2}} d \eta|y|\left|\phi^{\prime}(x-y \xi-i y \eta)\right|^{2} \\
& =\int_{-1}^{1} d \xi \int_{-2}^{-\frac{1}{2}} d \eta \iint_{Q^{*}\left(x_{0}, h\right)}|y|\left|\phi^{\prime}(x-y \xi-i y \eta)\right|^{2} d x d y \\
& =\int_{-1}^{1} d \xi \int_{-2}^{-\frac{1}{2}} d \eta \iint_{w\left(Q^{*}\left(x_{0}, h\right)\right)} \frac{|v|}{\eta^{2}}\left|\phi^{\prime}(u+i v)\right|^{2} d u d v \\
& \leq \int_{-1}^{1} d \xi \int_{-2}^{-\frac{1}{2}} \frac{d \eta}{\eta^{2}} \iint_{Q^{*}\left(x_{0}, 4 h\right)}|v|\left|\phi^{\prime}(u+i v)\right|^{2} d u d v \\
& =3 \iint_{Q^{*}\left(x_{0}, 4 h\right)}|v|\left|\phi^{\prime}(u+i v)\right|^{2} d u d v \\
& =3 \iint_{Q^{*}\left(x_{0}, 4 h\right)} \kappa_{\phi}(w) d u d v
\end{aligned}
$$

where we have used the change of variables $w=u+i v=x-\xi y-i \eta y$ which maps $Q^{*}\left(x_{0}, h\right)$ into $Q^{*}\left(x_{0}, 4 h\right)$. Consequently, $M_{\delta}\left(\lambda_{\phi^{\prime \prime}}\right) \lesssim M_{4 \delta}\left(\kappa_{\phi}\right)$, which implies that $\phi^{\prime \prime} \in \mathcal{B}\left(\mathbb{U}^{*}\right)$, and $\left\|\phi^{\prime \prime}\right\|_{\mathcal{B}} \lesssim\|\phi\|_{\text {BMOA }}$, while $\phi^{\prime \prime} \in \mathcal{B}_{0}\left(\mathbb{U}^{*}\right)$ if $\phi \in \operatorname{VMOA}\left(\mathbb{U}^{*}\right)$.

Conversely, suppose $\phi^{\prime \prime} \in \mathcal{B}\left(\mathbb{U}^{*}\right)$ so that $\phi^{\prime \prime} \in B\left(\mathbb{U}^{*}\right)$. It follows from (6.10) that

$$
|y|\left|\phi^{\prime}(x+i y)\right|^{2} \leq \int_{-\infty}^{y}\left|\phi^{\prime \prime}(x+i v)\right|^{2} v^{2} d v .
$$


Thus

$$
\begin{aligned}
& \iint_{Q^{*}\left(x_{0}, h\right)} \kappa_{\phi}(z) d x d y \lesssim \int_{x_{0}-h}^{x_{0}+h} d x \int_{-h}^{0} d y \int_{-\infty}^{y}\left|\phi^{\prime \prime}(x+i v)\right|^{2} v^{2} d v \\
& =\int_{x_{0}-h}^{x_{0}+h} d x \int_{-h}^{0} d y\left(\int_{-\infty}^{-h}\left|\phi^{\prime \prime}(x+i v)\right|^{2} v^{2} d v+\int_{-h}^{y}\left|\phi^{\prime \prime}(x+i v)\right|^{2} v^{2} d v\right) \\
& =\int_{x_{0}-h}^{x_{0}+h} d x\left(\int_{-h}^{0} d y \int_{-\infty}^{-h}\left|\phi^{\prime \prime}(x+i v)\right|^{2} v^{2} d v+\int_{-h}^{0} d v \int_{v}^{0}\left|\phi^{\prime \prime}(x+i v)\right|^{2} v^{2} d y\right) \\
& =\int_{x_{0}-h}^{x_{0}+h} d x \int_{-h}^{0} d y \int_{-\infty}^{-h}\left|\phi^{\prime \prime}(x+i v)\right|^{2} v^{2} d v \\
& \quad+\int_{x_{0}-h}^{x_{0}+h} d x \int_{-h}^{0} d v\left|\phi^{\prime \prime}(x+i v)\right|^{2}|v|^{3} .
\end{aligned}
$$

Consequently,

$$
\kappa_{\phi}\left(Q^{*}\left(x_{0}, h\right)\right) \lesssim\left\|\phi^{\prime \prime}\right\|_{B\left(\mathbb{U}^{*}\right)}^{2} h+\lambda_{\phi^{\prime \prime}}\left(Q^{*}\left(x_{0}, h\right)\right) \lesssim\left\|\phi^{\prime \prime}\right\|_{\mathcal{B}}^{2} h+\lambda_{\phi^{\prime \prime}}\left(Q^{*}\left(x_{0}, h\right)\right),
$$

which implies that $\phi \in \operatorname{BMOA}\left(\mathbb{U}^{*}\right)$ with $\|\phi\|_{\text {BMOA }} \lesssim\left\|\phi^{\prime \prime}\right\|_{\mathcal{B}}$.

Now suppose $\phi^{\prime \prime} \in \mathcal{B}_{0}\left(\mathbb{U}^{*}\right)$ so that $\phi^{\prime \prime} \in B_{0}\left(\mathbb{U}^{*}\right)$. Then for any $\epsilon>0$ there exists some $\delta_{0}>0$ such that

$$
|v|^{2}\left|\phi^{\prime \prime}(x+i v)\right|<\epsilon, \quad x+i v \in \mathbb{U}_{2, \delta_{0}}^{*} .
$$

Then, when $-\delta_{0}<h<0$, it follows from (7.2) that

$$
\kappa_{\phi}\left(Q^{*}\left(x_{0}, h\right)\right) \lesssim\left\|\phi^{\prime \prime}\right\|_{B\left(\mathbb{U}^{*}\right)}^{2} \frac{h^{2}}{\delta_{0}}+\epsilon^{2} h+\lambda_{\phi^{\prime \prime}}\left(Q^{*}\left(x_{0}, h\right)\right)
$$

which implies that $N_{0}\left(\kappa_{\phi}\right)=0$, that is, $\phi \in \operatorname{VMOA}\left(\mathbb{U}^{*}\right)$.

Examining the proof of Lemma 7.3, we find out that the following general result holds.

Proposition 7.4. Let $\phi$ be a holomorphic function on the lower half plane $\mathbb{U}^{*}$ such that $\lim _{y \rightarrow-\infty} \phi(x+i y)=0$ uniformly for $x \in \mathbb{R}$. For $\alpha>0$ set $\lambda_{1}(z)=$ $|\phi(z)|^{2}|y|^{\alpha}$ and $\lambda_{2}(z)=\left|\phi^{\prime}(z)\right|^{2}|y|^{\alpha+2}$. Then $\lambda_{1} \in C M\left(\mathbb{U}^{*}\right)$ if and only if $\lambda_{2} \in$ $C M\left(\mathbb{U}^{*}\right)$, and $N\left(\lambda_{1}\right) \asymp N\left(\lambda_{2}\right)$, while $\lambda_{1} \in C M_{0}\left(\mathbb{U}^{*}\right)$ if and only if $\lambda_{2} \in C M_{0}\left(\mathbb{U}^{*}\right)$.

Now we can prove the second main result of this paper.

Theorem 7.5.Let $\mu \in M(\mathbb{U})$ be given. Then $L(\mu) \in \operatorname{VMOA}\left(\mathbb{U}^{*}\right)$ if and only if $\mathcal{S}(\mu) \in \mathcal{B}_{0}\left(\mathbb{U}^{*}\right)$.

Proof. It follows from (6.6) that

$$
|\mathcal{S}(\mu)|^{2}|y|^{3} \lesssim\left|L^{\prime \prime}(\mu)\right|^{2}|y|^{3}+\left|L^{\prime}(\mu)\right|^{4}|y|^{3} \lesssim\left|L^{\prime \prime}(\mu)\right|^{2}|y|^{3}+\left|L^{\prime}(\mu)\right|^{2}|y|,
$$

which implies by Lemma 7.3 that $\mathcal{S}(\mu) \in \mathcal{B}_{0}\left(\mathbb{U}^{*}\right)$ whenever $L(\mu) \in \operatorname{VMOA}\left(\mathbb{U}^{*}\right)$.

Conversely, suppose $\mathcal{S}(\mu) \in \mathcal{B}_{0}\left(\mathbb{U}^{*}\right)$. We want to prove $L(\mu) \in \operatorname{VMOA}\left(\mathbb{U}^{*}\right)$, that is, $\left|L^{\prime}(\mu)(z)\right|^{2}|y| \in C M_{0}\left(\mathbb{U}^{*}\right)$, or equivalently, $\left|L^{\prime}(\mu)(\bar{z})\right|^{2} y \in C M_{0}(\mathbb{U}), z=x+i y \in \mathbb{U}$. We will use Proposition 3.1 to prove this. For any $\zeta=\xi+i \eta \in \mathbb{U}$, set

$$
\gamma_{\zeta}(z)=\frac{z-\zeta}{z-\bar{\zeta}}, \quad z=x+i y \in \mathbb{U}
$$


Then,

$$
\gamma_{\zeta}^{\prime}(z)=\frac{\zeta-\bar{\zeta}}{(z-\bar{\zeta})^{2}}, \quad N_{\gamma_{\zeta}}=\frac{\gamma_{\zeta}^{\prime \prime}(z)}{\gamma_{\zeta}^{\prime}(z)}=-\frac{2}{z-\bar{\zeta}} .
$$

Set $f(z)=\overline{f_{\mu}(\bar{z})}$ for $z \in \mathbb{U}$ and $f_{\zeta}=f \circ \gamma_{\zeta}^{-1}$ so that $f=f_{\zeta} \circ \gamma_{\zeta}$. Then

$$
N_{f}=N_{f_{\zeta} \circ \gamma_{\zeta}}=\left(N_{f_{\zeta}} \circ \gamma_{\zeta}\right) \gamma_{\zeta}^{\prime}+N_{\gamma_{\zeta}}=\left(N_{f_{\zeta}} \circ \gamma_{\zeta}-N_{\gamma_{\zeta}^{-1}} \circ \gamma_{\zeta}\right) \gamma_{\zeta}^{\prime} \text {. }
$$

Since $\mathcal{S}(\mu) \in \mathcal{B}_{0}\left(\mathbb{U}^{*}\right) \subset B_{0}\left(\mathbb{U}^{*}\right)$, Theorem 6.2 implies that $L(\mu) \in \mathbb{B}_{0}\left(\mathbb{U}^{*}\right)$. Thus, for $z=x+i y,\left|S_{f}(z)\right|^{2} y^{3} \in C M_{0}(\mathbb{U}), \lim _{y \rightarrow 0+}\left|S_{f}(z)\right| y^{2} \rightarrow 0$ and $\lim _{y \rightarrow 0+}\left|N_{f}(z)\right| y \rightarrow$ 0 uniformly for $x \in \mathbb{R}$. We need to show that $\left|N_{f}(z)\right|^{2} y \in C M_{0}(\mathbb{U})$, or equivalently by Proposition 3.1,

$$
\lim _{\eta \rightarrow 0+} \iint_{\mathbb{U}}\left|\gamma_{\zeta}^{\prime}(z)\right| y\left|N_{f}(z)\right|^{2} d x d y=0
$$

uniformly for $\xi \in \mathbb{R}$.

Firstly we have

$$
\begin{aligned}
& \lim _{\eta \rightarrow 0+} \iint_{\mathbb{U}}\left|\gamma_{\zeta}^{\prime}(z)\right| y\left|N_{f}(z)\right|^{2} d x d y \\
& =\frac{1}{2} \lim _{\eta \rightarrow 0+} \iint_{\mathbb{U}}\left(1-\left|\gamma_{\zeta}\right|^{2}\right)\left|\left(N_{f_{\zeta}} \circ \gamma_{\zeta}-N_{\gamma_{\zeta}^{-1}} \circ \gamma_{\zeta}\right) \gamma_{\zeta}^{\prime}\right|^{2} d x d y \\
& =\frac{1}{2} \lim _{\eta \rightarrow 0+} \iint_{\Delta}\left(1-|w|^{2}\right)\left|N_{f_{\zeta}}(w)-N_{\gamma_{\zeta}^{-1}}(w)\right|^{2} d u d v \\
& \lesssim \lim _{\eta \rightarrow 0+}\left(\left|N_{f_{\zeta}}(0)-N_{\gamma_{\zeta}^{-1}}(0)\right|^{2}+\iint_{\Delta}\left(1-|w|^{2}\right)^{3}\left|N_{f_{\zeta}}^{\prime}(w)-N_{\gamma_{\zeta}^{-1}}^{\prime}(w)\right|^{2} d u d v\right) \\
& =\lim _{\eta \rightarrow 0+}\left(\left|N_{f}(\zeta)\right|^{2} \eta^{2}+\iint_{\Delta}\left(1-|w|^{2}\right)^{3}\left|S_{f_{\zeta}}(w)+\frac{1}{2}\left(N_{f_{\zeta}}^{2}(w)-N_{\gamma_{\zeta}^{-1}}^{2}(w)\right)\right|^{2} d u d v\right) \\
& \lesssim \lim _{\eta \rightarrow 0+} \iint_{\mathbb{U}} y^{3}\left|\gamma_{\zeta}^{\prime}(z)\right|\left(\left|S_{f}(z)\right|^{2}+\left|N_{f}(z)-2 N_{\gamma_{\zeta}}(z)\right|^{2}\left|N_{f}(z)\right|^{2}\right) d x d y \\
& \lesssim \lim _{\eta \rightarrow 0+} \iint_{\mathbb{U}} y^{3}\left|\gamma_{\zeta}^{\prime}(z)\right|\left(\left|N_{f}(z)\right|^{2}+\left|N_{\gamma_{\zeta}}(z)\right|^{2}\right)\left|N_{f}(z)\right|^{2} d x d y .
\end{aligned}
$$

Here we have used the well-known result (see Theroem 4.28 in [Zhu])

$$
\iint_{\Delta}|\phi(w)|^{2}\left(1-|w|^{2}\right) d u d v \asymp|\phi(0)|^{2}+\iint_{\Delta}\left|\phi^{\prime}(w)\right|^{2}\left(1-|w|^{2}\right)^{3} d u d v
$$

for any function $\phi$ holomorphic in the unit disk. It remains to show

$$
\lim _{\eta \rightarrow 0+} \iint_{\mathbb{U}} y^{3}\left|\gamma_{\zeta}^{\prime}(z)\right|\left(\left|N_{f}(z)\right|^{2}+\left|N_{\gamma_{\zeta}}(z)\right|^{2}\right)\left|N_{f}(z)\right|^{2} d x d y=0 .
$$

For any $\epsilon>0$, choose some $y_{0}>0$ such that $\left|N_{f}(z)\right| y<\epsilon$ as $0<y<y_{0}$. Then, by (7.3) we have

$$
\begin{aligned}
& \iint_{0<y<y_{0}} y^{3}\left|\gamma_{\zeta}^{\prime}(z)\right|\left(\left|N_{f}(z)\right|^{2}+\left|N_{\gamma_{\zeta}}(z)\right|^{2}\right)\left|N_{f}(z)\right|^{2} d x d y \\
& \leq \epsilon^{2} \iint_{\mathbb{U}} y\left|\gamma_{\zeta}^{\prime}(z)\right|\left(\left|N_{f}(z)\right|^{2}+\left|N_{\gamma_{\zeta}}(z)\right|^{2}\right) d x d y \\
& \lesssim \epsilon^{2}\left(N\left(\kappa_{L(\mu)}\right)+\iint_{\mathbb{U}} \frac{y \eta}{\mid z-\bar{\zeta}^{4}} d x d y\right) .
\end{aligned}
$$


On the other hand, we have by (7.3) again that

$$
\iint_{y>y_{0}} y^{3}\left|\gamma_{\zeta}^{\prime}(z)\right|\left(\left|N_{f}(z)\right|^{2}+\left|N_{\gamma_{\zeta}}(z)\right|^{2}\right)\left|N_{f}(z)\right|^{2} d x d y \lesssim \iint_{y>y_{0}} \frac{\eta}{y|z-\bar{\zeta}|^{2}} d x d y .
$$

Noting that

$$
\begin{aligned}
\iint_{\mathbb{U}} \frac{y \eta}{|z-\bar{\zeta}|^{4}} d x d y & =\int_{0}^{+\infty} d y \int_{\mathbb{R}} \frac{y \eta}{|x+i y-\bar{\zeta}|^{4}} d x \\
& =\int_{0}^{+\infty} y \eta d y\left(\left.2 \pi i\left((z+i y-\bar{\zeta})^{-2}\right)^{\prime}\right|_{z=i y+\zeta}\right) \\
& =\frac{\pi}{2} \int_{0}^{+\infty} \frac{y \eta}{(y+\eta)^{3}} d y=\frac{\pi}{4}
\end{aligned}
$$

and

$$
\begin{aligned}
\iint_{y>y_{0}} \frac{\eta}{y|z-\bar{\zeta}|^{2}} d x d y & =\int_{y_{0}}^{+\infty} d y \int_{\mathbb{R}} \frac{\eta}{y|x+i y-\bar{\zeta}|^{2}} d x \\
& =\int_{y_{0}}^{+\infty} \frac{\eta}{y} d y\left(\left.2 \pi i(z+i y-\bar{\zeta})^{-1}\right|_{z=i y+\zeta}\right) \\
& =\pi \int_{y_{0}}^{+\infty} \frac{\eta}{y(y+\eta)} d y=\pi \ln \frac{y_{0}+\eta}{y_{0}}
\end{aligned}
$$

we find out that (7.6) follows from (7.7) and (7.8).

Theorem 7.6. $L: \mathcal{M}_{0}(\mathbb{U}) \rightarrow \operatorname{VMOA}\left(\mathbb{U}^{*}\right)$ is holomorphic.

Proof. Recall that the holomorphy of $L: \mathcal{M}(\mathbb{U}) \rightarrow \operatorname{BMOA}\left(\mathbb{U}^{*}\right)$ was proved in our paper [SW] (see Remark 1.1). It follows from Proposition 5.1 and Theorem 7.5 that $L$ maps $\mathcal{M}_{0}(\mathbb{U})$ into $\operatorname{VMOA}\left(\mathbb{U}^{*}\right)$. Thus $L: \mathcal{M}_{0}(\mathbb{U}) \rightarrow \operatorname{VMOA}\left(\mathbb{U}^{*}\right)$ is holomorphic.

Here we give a direct approach to the fact that $L$ maps $\mathcal{M}_{0}(\mathbb{U})$ into $\operatorname{VMOA}\left(\mathbb{U}^{*}\right)$. Let $\mu \in \mathcal{M}_{0}(\mathbb{U})$ be given so that $\lambda_{\mu} \in C M_{0}(\mathbb{U})$. Replacing $\mu$ with $\sigma(\mu)$ if necessary, we may assume that $\mu \in M_{0}(\mathbb{U})$ as well (see Theorem 4.3). For each $\delta>0$ let as before $\chi_{\delta}$ denote the characteristic function on the set $\mathbb{U}_{1, \delta}$, and $\mu_{\delta}=\mu \chi_{\delta}$. Then $\left\|\mu_{\delta}-\mu\right\|_{\infty} \rightarrow 0$ as $\delta \rightarrow 0+$. On the other hand, by Proposition 3.4 we obtain $\lim _{\delta \rightarrow 0+} N\left(\lambda_{\mu}-\lambda_{\mu} \chi_{\delta}\right)=0$. Noting that $\lambda_{\mu} \chi_{\delta}=\lambda_{\mu_{\delta}}$, we have $\lim _{\delta \rightarrow 0+} N\left(\lambda_{\mu-\mu_{\delta}}\right)=0$. Consequently, $\lim _{\delta \rightarrow 0+}\left\|\mu-\mu_{\delta}\right\|_{c}=0$. By continuity we have $\left\|L(\mu)-L\left(\mu_{\delta}\right)\right\|_{\text {BMOA }} \rightarrow 0$ as $\delta \rightarrow 0+$. On the other hand, for any $x_{0} \in \mathbb{R}$ and $h>0$, it follows from (6.5) that

$$
\iint_{Q^{*}\left(x_{0}, h\right)}\left|L^{\prime}\left(\mu_{\delta}\right)(z)\right|^{2}|y| d x d y \lesssim \int_{x_{0}-h}^{x_{0}+h} d x \int_{-h}^{0} \frac{|y|}{(y-\delta)^{2}} d y \lesssim \frac{h^{3}}{\delta^{2}}
$$

which implies that $\left|L^{\prime}\left(\mu_{\delta}\right)(z)\right|^{2}|y| \in C M_{0}\left(\mathbb{U}^{*}\right)$, that is, $L\left(\mu_{\delta}\right) \in \operatorname{VMOA}\left(\mathbb{U}^{*}\right)$. Since $\operatorname{VMOA}\left(\mathbb{U}^{*}\right)$ is closed in $\operatorname{BMOA}\left(\mathbb{U}^{*}\right)$, we obtain $L(\mu) \in \operatorname{VMOA}\left(\mathbb{U}^{*}\right)$.

\section{Quasisymmetric homeomorphism model of $T_{v}$}

Each of the Teichmüller spaces $T, T_{0}, T_{b}$ and $T_{v}$ has a quasisymmetric homeomorphism model, namely, $T=\mathrm{QS}(\mathbb{R}) / \operatorname{Aff}(\mathbb{R}), T_{0}=\mathrm{S}(\mathbb{R}) / \operatorname{Aff}(\mathbb{R}), T_{b}=\mathrm{SQS}(\mathbb{R}) / \operatorname{Aff}(\mathbb{R})$, and $T_{v}=\mathrm{SS}(\mathbb{R}) / \operatorname{Aff}(\mathbb{R})$. Now each of these four Teichmüller spaces has been endowed with the standard complex Banach manifold structure via the Bers embedding. Furthermore, the BMO-Teichmüller space $T_{b}$ has another real Banach manifold structure (see [FHS2] and Theorem 8.1 below). To make this precise, we recall some basic definitions on BMO functions. 
Let $\Gamma$ be a locally rectifiable Jordan curve in the complex plane. We denote by $\mathrm{BMO}(\Gamma)$ the space of locally integrable functions $u$ on $\Gamma$ of bounded mean oscillation (see [FS], [Gar]), namely,

$$
\|u\|_{\mathrm{BMO}} \doteq \sup \frac{1}{|I|} \int_{I}\left|u(\zeta)-u_{I}\right||d \zeta|<+\infty
$$

where the supremum is taken over all finite sub-arcs $I$ of $\Gamma$, while $u_{I}$ is the average of $u$ on the interval $I$, namely,

$$
u_{I}=\frac{1}{|I|} \int_{I} u(\zeta)|d \zeta|
$$

If $u$ also satisfies the condition

$$
\lim _{|I| \rightarrow 0} \frac{1}{|I|} \int_{I}\left|u(\zeta)-u_{I}\right||d \zeta|=0
$$

we say $u$ has vanishing mean oscillation and belongs to the space $\operatorname{VMO}(\Gamma)$ (see [Gar], $[\mathrm{Sa}])$. In the following, we denote by $\mathrm{BMO}_{\mathbb{R}}(\Gamma)\left(\operatorname{VMO}_{\mathbb{R}}(\Gamma)\right)$ the set of all real-valued BMO (VMO) functions.

Recall that a strongly quasisymmetric homeomorphism $h$ is locally absolutely continuous such that $\log h^{\prime}$ is a BMO function. The following result was proved in our previous papers [SW] and [FHS2].

Theorem 8.1 The correspondence $h \mapsto \log h^{\prime}$ induces a homeomorphism $\Psi$ from $T_{b}$ onto a connected open subset of $\mathrm{BMO}_{\mathbb{R}}(\mathbb{R}) / \mathbb{R}$.

Now we continue to consider to what extent one can extend Theorem 7.2 to the VMO-Teichmüller space and prove the third main result of this paper.

Theorem 8.2. Let $h$ be a strongly symmetric homeomorphism on the real line. Then $h$ is strongly quasisymmetric such that $\log h^{\prime}$ is a VMO function.

Proof. Suppose $h$ is a strongly symmetric homeomorphism on the real line. Then $h$ can be extended a quasiconformal mapping $f$ to the upper half plane $\mathbb{U}$ with Beltrami coefficient $\mu \in \mathcal{M}_{0}(\mathbb{U})$. Clearly, $h$ is strongly quasisymmetric such that $\log h^{\prime} \in \mathrm{BMO}(\mathbb{R})$. As in the proof of Theorem 7.6, we may assume that $\mu \in M_{0}(\mathbb{U})$ as well, and for each $\delta>0$ let $\mu_{\delta}=\mu \chi_{\delta}$, where $\chi_{\delta}$ is the characteristic function on the set $\mathbb{U}_{1, \delta}$. Then $\lim _{\delta \rightarrow 0+}\left\|\mu-\mu_{\delta}\right\|_{c}=0$. By Theorem 8.1 we have $\left\|\log h_{\delta}^{\prime}-\log h^{\prime}\right\|_{\text {BMO }} \rightarrow 0$ as $\delta \rightarrow 0+$, where $h_{\delta}=\left.f^{\mu_{\delta}}\right|_{\mathbb{R}}$.

On the other hand, we consider

$$
g_{\delta}(x+i y)= \begin{cases}f^{\mu_{\delta}}(x+i y), & 0 \leq y<\delta, \\ f^{\mu_{\delta}}(x-i y), & -\delta<y \leq 0 .\end{cases}
$$

Then $g_{\delta}$ is conformal in the horizontal strip $\{z=x+i y:|y|<\delta\}$ with $\left.g_{\delta}\right|_{\mathbb{R}}=h_{\delta}$. We conclude by the following Lemma 8.3 that $\log h_{\delta}^{\prime}=\left.\log g_{\delta}^{\prime}\right|_{\mathbb{R}}$ is Lipschitz continuous on the real line. Recall that $\operatorname{VMO}(\mathbb{R})$ is the closure under the BMO-norm of the set of all uniformly continuous BMO functions on the real line (see [Gar], [Sa]), we conclude by $\lim _{\delta \rightarrow 0+}\left\|\log h_{\delta}^{\prime}-\log h^{\prime}\right\|_{\text {BMO }}=0$ that $\log h^{\prime} \in \operatorname{VMO}(\mathbb{R})$.

Lemma 8.3. Let $g$ be a conformal mapping on the horizontal strip $S_{\delta}=\{z=$ $x+i y:|y|<\delta\}$. Then it holds that

$$
\left|\frac{2 \delta}{\pi} N_{g}(z)-1\right| \cos \left(\frac{\pi}{2 \delta} y\right) \leq 3, \quad z=x+i y \in S_{\delta}
$$

In particular, $\log g^{\prime}$ is Lipschitz continuous on each horizontal line in $S_{\delta}$. 
Proof. Set $f(\zeta)=g\left(2 \pi^{-1} \delta \log \zeta-i \delta\right)$. Then $f$ is a conformal mapping on the upper half plane $\mathbb{U}$. By Proposition 6.1 we obtain $\left|N_{f}(\zeta)\right| \eta \leq 3$ for $\zeta=\xi+i \eta \in \mathbb{U}$. Noting that

$$
N_{f}(\zeta)=\frac{2 \delta}{\pi \zeta} N_{g}\left(2 \pi^{-1} \delta \log \zeta-i \delta\right)-\frac{1}{\zeta}
$$

we obtain

$$
\left|\frac{2 \delta}{\pi \zeta} N_{g}\left(2 \pi^{-1} \delta \log \zeta-i \delta\right)-\frac{1}{\zeta}\right| \eta \leq 3 .
$$

For $z=x+i y \in S_{\delta}$, letting $\zeta=i \exp \left(\frac{\pi}{2 \delta} z\right) \in \mathbb{U}$ we obtain (8.4). When $g(z)=$ $\exp \left(-\frac{\pi}{\delta} z\right)$, we have $N_{g}(z)=-\frac{\pi}{\delta}$, which implies that the estimate (8.4) is sharp. On each horizontal line in $S_{\delta}$ we conclude by (8.4) that $N_{g}=\left(\log g^{\prime}\right)^{\prime}$ is bounded, which implies that $\log g^{\prime}$ is Lipschitz continuous.

Remark 8.4. It is not clear whether the converse of Theorem 8.2 is true (see Problem 9.1 below). Examining the proof of Proposition 4.2 in [Se2], we may find out that an increasing homeomorphism $h$ on the real line is strongly symmetric if $h$ is locally absolutely continuous such that $\log h^{\prime}$ is a VMO function with small BMO norm $\left\|\log h^{\prime}\right\|_{\text {BMO }}$.

Here is an appropriate place to relate a well-known result of Carleson [Ca]. For a Beltrami coefficient $\mu \in M(\mathbb{U})$, one may consider $k_{\delta}(\mu)=\left\|\left.\mu\right|_{\mathbb{U}_{2, \delta}}\right\|_{\infty}$. Then $\mu \in$ $M_{0}(\mathbb{U})$ if and only if $\lim _{\delta \rightarrow 0+} k_{\delta}(\mu)=0$. Carleson [Ca] proved that $h=\left.f^{\mu}\right|_{\mathbb{R}}$ is locally absolutely continuous with $h^{\prime} \in L_{\mathrm{loc}}^{2}$ if $\mu$ satisfies the following condition

$$
\int_{0} \frac{k_{\delta}^{2}(\mu)}{\delta} d \delta<+\infty
$$

Later, Anderson-Becker-Lesley [ABL] extended this to the following result. We give a fast approach to this result by means of Theorem 8.2.

Corollary 8.5. Let $\mu \in M(\mathbb{U})$ satisfy the condition (8.5). Then $h=\left.f^{\mu}\right|_{\mathbb{R}}$ is locally absolutely continuous such that $\log h^{\prime}$ is a VMO function on any finite interval $[a, b]$ on the real line.

Proof. Let $\mu \in M(\mathbb{U})$ satisfy the condition (8.5). Fix some $\delta>0$. Set as before $\mu_{\delta}=\mu \chi_{\delta}$, where $\chi_{\delta}$ is the characteristic function on the set $\mathbb{U}_{1, \delta}$, and $\hat{\mu}_{\delta}=\mu-\mu_{\delta}$. Then $\hat{\mu}_{\delta} \in \mathcal{M}_{0}(\mathbb{U})$, or equivalently, $\lambda_{\hat{\mu}_{\delta}} \in C M_{0}(\mathbb{U})$. In fact,

$$
\begin{aligned}
\lambda_{\hat{\mu}_{\delta}}\left(Q\left(x_{0}, h\right)\right) & =\int_{x_{0}-h}^{x_{0}+h} d x \int_{0}^{h} \frac{\left|\hat{\mu}_{\delta}(x+i y)\right|^{2}}{y} d y \\
& \leq \int_{x_{0}-h}^{x_{0}+h} d x \int_{0}^{\min (h, \delta)} \frac{k_{y}^{2}(\mu)}{y} d y=2 h \int_{0}^{\min (h, \delta)} \frac{k_{y}^{2}(\mu)}{y} d y
\end{aligned}
$$

which implies that $\lambda_{\hat{\mu}_{\delta}} \in C M_{0}(\mathbb{U})$ by the assumption (8.5). Therefore, $\hat{h}_{\delta}=\left.f^{\hat{\mu}_{\delta}}\right|_{\mathbb{R}}$ is strongly symmetric, which implies by Theorem 8.2 that $\hat{h}_{\delta}$ is strongly quasisymmetric and $\log \hat{h}_{\delta}^{\prime} \in \operatorname{VMO}(\mathbb{R})$.

Now we set $g_{\delta}=f^{\mu} \circ\left(f^{\hat{\mu}_{\delta}}\right)^{-1}$ so that $f^{\mu}=g_{\delta} \circ f^{\hat{\mu}_{\delta}}$. Set $D_{\delta}=f^{\hat{\mu}_{\delta}}\left(\mathbb{U}_{2, \delta}\right)$, $D_{\delta}^{*}=\overline{f^{\mu_{\delta}}}\left(\mathbb{U}_{2, \delta}^{*}\right)$, and $S_{\delta}=D_{\delta} \cup \mathbb{R} \cup D_{\delta}^{*}$. Then $g_{\delta}$ is a quasiconformal mapping of $\mathbb{U}$ onto itself and is conformal in $D_{\delta}$. Clearly, $\left.g_{\delta}\right|_{D_{\delta}}$ can be extended a conformal mapping to $S_{\delta}$ by reflection, which implies that $g_{\delta}$ is real analytic on the real line, in particular, $\left.\log g_{\delta}^{\prime}\right|_{\mathbb{R}}$ is a VMO function on any finite interval $[a, b]$ on the real line. 
Then, $h=\left.g_{\delta}\right|_{\mathbb{R}} \circ \hat{h}_{\delta}$ is locally absolutely continuous such that $h^{\prime}=\left(\left.g_{\delta}^{\prime}\right|_{\mathbb{R}} \circ \hat{h}_{\delta}\right) \hat{h}_{\delta}^{\prime}$. Consequently,

$$
\log h^{\prime}=\log \left(\left.g_{\delta}^{\prime}\right|_{\mathbb{R}} \circ \hat{h}_{\delta}\right)+\log \hat{h}_{\delta}^{\prime}
$$

which implies that $\log h^{\prime}$ is a VMO function on any finite interval $[a, b]$ on the real line (see Lemma in $[\mathrm{ABL}]$ ).

Remark 8.6. It is well-known that a quasisymmetric homeomorphism need not be locally absolutely continuous (see [BA]). An important problem of long time has been to determine when a quasisymmetric homeomorphism is locally absolutely continuous. Carleson [Ca] initiated such an investigation, giving the sufficient condition (8.5) on the dilatation of a quasiconformal self-mapping of the upper half plane to have locally absolutely continuous boundary values. Since then there have appeared many works to deal with this problem (see [ABL], [AZ], [Dy], [FKP], [Ma], [Se2]). For example, a much satisfactory answer is contained in Theorem 7.2. Very recently, we established several results on the Weil-Petersson Teichmüller space which are related to this problem (see [Sh], [ST], [STW], [SWu], [WHS]). Theorem 8.2 can be considered as a new result on this problem.

\section{Additional remarks and problems}

We end the paper with several remarks and problems related to strongly symmetric homeomorphisms.

By Theorem 8.2 it is natural to consider the following sub-class of quasisymmetric homeomorphisms

$$
\widetilde{\mathrm{SS}}(\mathbb{R})=\left\{h \in \mathrm{SQS}(\mathbb{R}): \log h^{\prime} \in \mathrm{VMO}(\mathbb{R})\right\}
$$

and the sub-space $\tilde{T}_{v}=\widetilde{\mathrm{SS}}(\mathbb{R}) / \operatorname{Aff}(\mathbb{R})$ of the universal Teichmüller space. Then $\mathrm{SS}(\mathbb{R}) \subset \widetilde{\mathrm{SS}}(\mathbb{R})$, and $T_{v} \subset \tilde{T}_{v}$ by Theorem 8.2. On the other hand, examining the proof of Lemma 3.3 in our paper $[\mathrm{Sh}]$, we find out that $\widetilde{\mathrm{SS}}(\mathbb{R}) \subset \mathrm{S}(\mathbb{R})$ so that $\tilde{T}_{v} \subset T_{0}$.

Problem 9.1. Determine whether it is true that $\mathrm{SS}(\mathbb{R})=\widetilde{\mathrm{SS}}(\mathbb{R})$. If this were true, we would have an intrinsic characterization for strongly symmetric homeomorphisms without using quasiconformal extensions, namely, a homeomorphism $h \in \operatorname{Hom}^{+}(\mathbb{R})$ is strongly symmetric if and only if $h$ is strongly quasisymmetric such that $\log h^{\prime} \in \operatorname{VMO}(\mathbb{R})$.

Recall that $\Psi$ is the homeomorphism from $T_{b}$ onto a connected open subset of $\mathrm{BMO}_{\mathbb{R}}(\mathbb{R}) / \mathbb{R}$ induced by the correspondence $h \mapsto \log h^{\prime}$ (see Theorem 8.1). Under the mapping $\Psi$, it holds by definition that $\Psi\left(\tilde{T}_{v}\right)=\Psi\left(T_{b}\right) \cap\left(\operatorname{VMO}_{\mathbb{R}}(\mathbb{R}) / \mathbb{R}\right)$. Since $\Psi\left(T_{b}\right)$ is a connected open subset of $\mathrm{BMO}_{\mathbb{R}}(\mathbb{R}) / \mathbb{R}$, we conclude that $\Psi\left(\tilde{T}_{v}\right)$ is a connected open subset of $\operatorname{VMO}_{\mathbb{R}}(\mathbb{R}) / \mathbb{R}$, which enables one to endow $\tilde{T}_{v}$ with a real Banach manifold structure.

Problem 9.2. Determine whether or not $\Psi\left(T_{v}\right)$ is an open subset of $\operatorname{VMO}_{\mathbb{R}}(\mathbb{R}) / \mathbb{R}$. If so, we would be able to endow the VMO-Teichmüller space $T_{v}$ with another real Banach manifold structure, which is topologically equivalent to the standard complex Banach manifold structure via the Bers embedding (see Theorem 2.1).

Acknowledgements. I would like to thank Wei Huaying for helpful discussions and for letting me know her recent work [WM3] with K. Matsuzaki, where they proved that $\widetilde{S S}(\mathbb{R}) \subset \mathrm{SS}(\mathbb{R})$. I also thank the referee for a very careful reading of the manuscript and for useful comments. 


\section{References}

[Ah] Ahlfors, L. V.: Quasiconformal reflections. - Acta Math. 109, 1963, 291-301.

[ABL] Anderson, J. M., J. Becker, and F. D. Lesley: On the boundary correspondence of asymptotically conformal automorphisms. - J. London Math. Soc. 38, 1988, 453-462.

[AG] Astala, K., and F. W. Gehring: Injectivity, the BMO norm and the universal Teichmüller space. - J. Anal. Math. 46, 1986, 16-57.

[AZ] Astala, K., and M. Zinsmeister: Teichmüller spaces and BMOA. - Math. Ann. 289, 1991, 613-625.

[BA] Beurling, A., and L. V. Ahlfors: The boundary correspondence under quasiconformal mappings. - Acta Math. 96, 1956, 125-142.

[BJ] Bishop, C., and P. Jones: Harmonic measure, $L^{2}$ estimates and the Schwarzian derivative. - J. Anal. Math. 62, 1994, 77-113.

[Ca] Carleson, L.: On mappings, conformal at the boundary. - J. Anal. Math. 19, 1967, 1-13.

[CF] Colfman, R. R., and C. Fefferman: Weighted norm inequalities for maximal functions and singular integrals. - Studia Math. 51, 1974, 241-250.

$[\mathrm{Cu}]$ CUI, G.: Integrably asymptotic affine homeomorphisms of the circle and Teichmüller spaces. - Sci. China Ser. A 43, 2000, 267-279.

[CZ] Cui, G., and M. Zinsmeister: BMO-Teichmüller spaces. - Illinois J. Math. 48, 2004, $1223-1233$.

[Da] Dahlberg, B.: On the absolute continuity of elliptic measures. - Amer. J. Math. 108, 1986, 1119-1138.

[DE] Douady, A., and C. J. EARLE: Conformally natural extension of homeomorphisms of the circle. - Acta Math. 157, 1986, 23-48.

[Dy] Dynkin, E.: Estimates for asymptotically conformal mappings. - Ann. Acad. Sci. Fenn. Math. 22, 1997, 275-304-

[EMS] Earle, C. J., V. Markovic, and D. Saric: Barycentric extension and the Bers embedding for asymptotic Teichmüller space. - Contemp. Math. 311, 2002, 87-105.

[EN] EARLe, C. J., and S. NAG: Conformally natural reflections in Jordan curves with applications to Teichmüller spaces. - In: Holomorphic Functions and Moduli II, MSRI Publications 11, Springer-Verlag, 1988, 179-194.

[FHS1] FAn, Y., Y. Hu, and Y. SHEn: A note on BMO map induced by strongly quasisymmetric homeomorphism. - Proc. Amer. Math. Soc. 145, 2017, 2505-2512

[FHS2] FAn, Y., Y. Hu, and Y. Shen: On strongly quasisymmetric homeomorphisms. - Ann. Acad. Sci. Fenn. Math. 42, 2017, 921-930.

[FKP] Fefferman, R., C. Kenig, and J. Pipher: The theory of weights and the Dirichlet problems for elliptic equations. - Ann. Math. 134, 1991, 65-124.

[FS] Fefferman, C., and E. Stein: $H^{p}$ spaces of several variables. - Acta Math. 129, 1972, $137-193$.

[Ga] Gardiner, F. P.: Teichmüller theory and quadratic differentials. - Wiley-Interscience, New York, 1987.

[GL] Gardiner, F. P., and N. Lakic: Quasiconformal Teichmüller theory. - Math. Surveys Monogr. 76, Amer. Math. Soc., Providence, RI, 2000.

[GS] Gardiner, F. P., and D. Sullivan: Symmetric structures on a closed curve. - Amer. J. Math. 114, 1992, 683-736.

[Gar] Garnett, J. B.: Bounded analytic functions. - Academic Press, New York, 1981.

[Ha] Hamilton, D. E.: BMO and Teichmüller spaces. - Ann. Acad. Sci. Fenn. Math. 14, 1989, 213-224. 
[HS] Hu, Y., and Y. Shen: On quasisymmetric homeomorphisms. - Israel J. Math. 191, 2012, 209-226.

[HWS] Hu, Y., L. Wu, and Y. Shen: On symmetric homeomorphisms on the real line. - Proc. Amer. Math. Soc. 146, 2018, 4255-4263.

[Jo] Jones, P. W: Homeomorphisms of the line which preserve BMO. - Ark. Math. 21, 1983, 229-231.

[Le] Lehto, O.: Univalent functions and Teichmüller spaces. - Springer-Verlag, New York, 1986.

[Ma] Macmanus, P.: Quasiconformal mappings and Ahlfors-David curves. - Trans. Amer. Math. Soc. 343, 1994, 853-881.

[Mat] Matsuzaki, K.: The universal Teichmüller space and diffeomorphisms of the circle with Hölder continuous derivatives. - In: Handbook of group action (vol. 1), Advanced Lectures in Mathematics 31, Higher Education Press and International Press, 333-372.

[Na] NAG, S.: The complex analytic theory of Teichmüller spaces. - Wiley-Interscience, 1988.

[Pa] PARTYKa, D.: Eigenvalues of quasisymmetric automorphisms determined by VMO functions. - Ann. Univ. Mariae Curie-Sklodowska Sect. A 52, 1998, 121-135.

[Po] Pommerenke, Ch.: Univalent functions. - Vandenhoeck and Ruprecht, 1975.

[Sa] Sarason, D.: Functions of vanishing mean oscillation. - Trans. Amer. Math. Soc. 207, 1975, 391-405.

[Se1] Semmes, S.: The Cauchy integral, chord-arc curves, and quasiconformal mappings. - In: Proc. Bieberbach Conf. (Purdue University, 1985) (edited by A. Baernstein, P. Duren, A. Marden, and D. Drasin), Math. Surveys 21, Amer. Math. Soc., Providence, R.I., 1986.

[Se2] Semmes, S.: Quasiconformal mappings and chord-arc curves. - Trans. Amer. Math. Soc. 306, 1988, 233-263.

[Sh] Shen, Y.: Weil-Peterssen Teichmüller space. - Amer. J. Math. 140, 2018, 1041-1074.

[ST] Shen, Y., and S. TANG: Weil-Petersson Teichmüller space II: smoothness of flow curves of $H^{\frac{3}{2}}$-vector fields. - Adv. Math. 359, 2020, 106891.

[STW] Shen, Y., S. TAng, and L. Wu: Weil-Petersson and little Teichmüller spaces on the real line. - Ann. Acad. Sci. Fenn. Math. 43, 2018, 935-943.

[SW] Shen, Y., and H. WeI: Universal Teichmüller space and BMO. - Adv. Math. 234, 2013, $129-148$.

[SWu] Shen, Y., and L. Wu: Weil-Petersson Teichmüller space III: dependence of Riemann mappings for Weil-Petersson curves. - Math. Ann. 381, 2021, 875-904.

[TT] Takhtajan, L., and L.-P. TeO: Weil-Petersson metric on the universal Teichmüller space. - Mem. Amer. Math. Soc. 183:861, 2006.

[TS] TAng, S., and Y. Shen: Integrable Teichmüller space. - J. Math. Anal. Appl. 465, 2018, 658-672.

[TWS] Tang, S., H. WeI, and Y. Shen: Douady-Earle extension and the contractibility of the VMO-Teichmüller space. - J. Math. Anal. Appl. 442, 2016, 376-384.

[We] Wei, H.: A note on the BMO-Teichmüller space. - J. Math. Anal. Appl. 435, 2016, 746-753.

[WM1] Wei, H., and K. Matsuzaki: Teichmüller spaces of generalized symmetric homeomorphisms. - Proc. Amer. Math. Soc. Ser. B 7, 2020, 52-66.

[WM2] Wei, H., and K. Matsuzaki: BMO Teichmüller spaces and their quotients with complex and metric structures. - arXiv: 1811.08785.

[WM3] Wei, H., and K. Matsuzaki: Beurling-Ahlfors extension by heat kernel, $A^{\infty}$-weights for VMO, and vanishing Carleson measures. - Bull. London. Math. Soc. 53, 2021, 723-739.

[WS] WeI, H., and Y. Shen: On the tangent space to the BMO-Teichmüller space. - J. Math. Anal. Appl. 419, 2014, 715-726. 
[WZ] Wei, H., and M. Zinsmeister: Carleson measures and chord-arc curves. - Ann. Acad. Sci. Fenn. Math. 43, 2018, 669-683.

[WHS] Wu, L., Y. Hu, and Y. Shen: Weil-Petersson Teichmüller space revisited. - J. Math. Anal. Appl. 491, 2020, 124304.

[Zh] Zhно, R.: Distances from Bloch functions to some Möbius invariant spaces. - Ann. Acad. Sci. Fenn. Math. 33, 2008, 303-313.

[Zhu] ZHu, K.: Operator theory in function spaces. Second edition. - Math. Surveys Monogr. 138, Amer. Math. Soc., Providence, RI, 2007.

[Zu] Zuravlev, I. V.: A model of the universal Teichmüller space. - Sib. Math. Z. 27, 1986, 75-82 (in Russian).

Received 7 November 2021 • Accepted 19 March $2021 \bullet$ Published online 29 November 2021

Yuliang Shen

Soochow University

Department of Mathematics

Suzhou 215006, P. R. China

ylshen@suda.edu.cn 